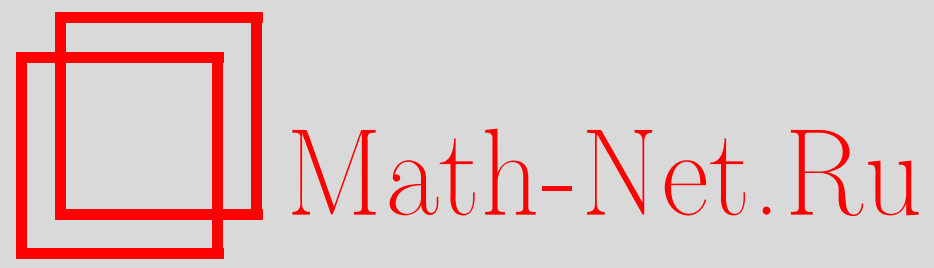

Б. А. Погорелов, М. А. Пудовкина, Комбинаторная характеризация XL-слоев, Матем. вопр. криптогр., 2013, том 4, выпуск 3, 99-129

DOI: https://doi.org/10.4213/mvk94

Использование Общероссийского математического портала Math-Net.Ru подразумевает, что вы прочитали и согласны с пользовательским соглашением

http://www . mathnet.ru/rus/agreement

Параметры загрузки:

IP : 54.198 .67 .100

26 апреля 2023 г., 13:25:24 


\title{
Комбинаторная характеризация XL-слоев
}

\author{
Б. А. Погорелов ${ }^{1}$, М. А. Пудовкина ${ }^{2}$ \\ ${ }^{1}$ Академия криптографии Российской Федерации, Москва \\ ${ }^{2}$ Национальный исследовательский ядерный университет (МИФИ), Москва
}

Получено 11.IV.2012

Рассматриваются различные комбинаторно-структурные свойства подгруппы аффинной группы, порожденной преобразованиями слоя наложения ключа (всеми сдвигами) и линейным слоем (в случае приводимости соответствующей матрицы). В частности, описаны свойства графов орбиталов этой подгруппы.

Ключевые слова: орбитал, граф орбитала, метрика, дистанционно-транзитивный граф, дистанционно-регулярный граф

\section{Combinatorial characterization of XL-layers}

\section{B. A. Pogorelov ${ }^{1}$, M. A. Pudovkina ${ }^{2}$}

${ }^{1}$ Academy of Cryptography of the Russian Federation, Moscow

${ }^{2}$ National Nuclear Research University, Moscow

\begin{abstract}
We consider combinatorial and structural properties of an affine subgroup generated by key addition and linear layer, i.e. by all shifts and by the multiplication on a reducible matrix. In particular, we describe properties of orbital graphs of this subgroup. Key words: orbital, orbital graph, metric, distance-transitive graph, distance-regular graph

Citation: Mathematical Aspects of Cryptography, 2013, vol. 4, no. 3, pp. 99-129 (Russian).
\end{abstract}




\section{1. Введение}

Алгоритмы блочного шифрования реализуются итеративным применением в раундах более простых (базовых) преобразований, которые должны обеспечивать свойства перемешивания, рассеивания и усложнения. Для обеспечения данных свойств в раунде обычно используются преобразования (слои) трех типов: аддитивное наложение ключа, нелинейные преобразования над отдельными частями блока текста (s-боксы) и линейные преобразования $g$, переставляющие между собой части этого блока. Схемы, реализующие подобные алгоритмы, получили название SP-сетей. В последнее время перестановку отдельных блоков стали заменять более общими линейными или аффинными преобразованиями векторных пространств над полем $G F(2)$. Блочные шифрсистемы с таким построением раундовых преобразований и побитным подмешиванием раундового ключа в каждом раунде называют XSL-сетями. В частности, по такому принципу реализован алгоритм блочного шифрования AES.

В ряде случаев линейные преобразования, обеспечивающие хорошее рассеивание, являются приводимыми. Например, шифрсистемы ARIA, Camellia, MIBS имеют линейные преобразования с характеристическими многочленами над полем $G F(2)$ вида $(x+1)^{r}$ для некоторого натурального числа $r$. Приводимыми являются и подстановочные матрицы, используемые в SP-сетях. Это приводит к импримитивности подгруппы $C(g)$ аффинной группы $A G L_{n}(2)$, порожденной преобразованиями слоя наложения ключа (т. е. всеми сдвигами) и приводимой матрицей $g \in G L_{n}(2)$. При этом систем импримитивности, частично упорядоченных по вложению, может быть довольно много. Системы импримитивности сохраняются линейным слоем и слоем наложения ключа. Поэтому обеспечивать необходимое «рассеивание» блоков импримитивности в алгоритме шифрования могут только слои $s$-боксов.

В работе рассматриваются различные комбинаторно-структурные свойства группы $C(g)$. Описаны свойства графов орбиталов группы $C(g)$ и рассмотрены метрики как кратчайшие расстояния между вершинами в графе. В частности, в терминах характеристического или минимального многочленов $g$ приведены условия: связности графов орбиталов, их изоморфизма, примитивности и 2-транзитививности группы $C(g)$. Указаны условия дистанционной транзитивности и дистанционной регулярности графов орбиталов.

Рассмотрены также свойства графов орбиталов группы $C(g)$ в двух случаях: когда $g$ лежит в небольших надгруппах подстановочных групп и когда $g$ берется из унитреугольной группы $U T_{n}, n=2^{m}$. В этих случаях группа $C(g)$ 
обладает выраженными геометрическими свойствами. В этом смысле полученные результаты созвучны работам [4, 6], где рассмотрены свойства графов орбиталов надгрупп группы Джевонса и их метрики. Приведены условия на орбиталы, при которых соответствующие метрики изоморфны метрике Хемминга (на пространстве $V_{m}, m \leq n$ ), а также их надметрикам или подметрикам. Описаны метрики и другие характеристики графов орбиталов группы $C(g)$ для некоторых циркулянтных матриц $g$ и матриц блочных шифрсистем.

\section{2. Основные обозначения}

Пусть: $\mathbb{N}\left(\mathbb{N}_{0}\right)$ - множество всех натуральных (целых неотрицательных) чисел; $|A|$ - мощность множества $A ; V_{n}$ - векторное пространство размерности $n \geq 2$ над полем $G F(2) ; \varepsilon_{i}=(0, \ldots, 0,1, \underbrace{0, \ldots, 0}_{i}) \in V_{n}, i=0, \ldots, n-1$; $G L_{n}\left(A G L_{n}\right)$ - полная линейная (аффинная) группа над $G F(2) ; V_{n} / W-$ фактор-пространство пространства $V_{n}$ по подпространству $W ; X^{\times}=X \backslash\{\overrightarrow{0}\}$; $a(x) \mid b(x)$ - многочлен $a(x)$ делит многочлен $b(x) ; \operatorname{deg} b(x)$ - степень многочлена $b(x) ; u_{g}(x)\left(m_{g}(x)\right)$ - характеристический (минимальный) многочлен преобразования $g \in G L_{n}(2) ; m_{\gamma, g}(x)$ - минимальный многочлен вектора $\gamma \in$ $V_{n}^{\times}$относительно преобразования $g \in G L_{n}(2) ; d_{g}-$ число орбит группы $\langle g\rangle$ на $V_{n} ; A \oplus B=\{\alpha \oplus \beta \mid \alpha \in A, \beta \in B\}$, где $A, B \subseteq V_{n} ; \Delta_{i}^{(n)}$ - множество всех векторов из $V_{n}$ веса $i \in\{0, \ldots, n\} ; h_{\alpha}: \beta \rightarrow \beta \oplus \alpha$ - преобразование-сдвиг пространства $V_{n}$ на вектор $\alpha \in V_{n} ; g_{\alpha}=g h_{\alpha}, H_{n}=\left\{h_{\alpha}: V_{n} \rightarrow V_{n} \mid \alpha \in V_{n}\right\}-$ группа сдвигов; $\operatorname{diag}\left(g_{1}, \ldots, g_{p}\right)$ - клеточно-диагональная матрица, на диагонали которой находятся матрицы $g_{1}, \ldots, g_{p} ; C(g)=\left\langle g_{\alpha} \mid \alpha \in V_{n}\right\rangle=\langle g\rangle H_{n}-$ подгруппа аффинной группы; $S(X)$ - множество всех подстановок на $X ; S_{n}=$ $=S(X)$ при $|X|=n ; \alpha^{g}=\alpha g-$ образ элемента $\alpha \in X$ при действии на него подстановкой $g \in S(X) ; \alpha^{G}=\left\{\alpha^{g} \mid g \in G\right\}, G \leq S(X) ; S_{2} \uparrow S_{n}=H_{n} S_{n}-$ группа Джевонса, совпадающая с группой экспоненцирования; $\chi_{n}(\alpha, \beta)-$ расстояние Хемминга между векторами $\alpha, \beta \in V_{n} ; G_{1} \approx G_{2}-$ подобие групп $G_{1}, G_{2} ; \bar{\Gamma}_{1} \cong \bar{\Gamma}_{2}\left(\mu_{1} \cong \mu_{2}\right)-$ изоморфизм графов $\bar{\Gamma}_{1}, \bar{\Gamma}_{2}$ (метрик $\left.\mu_{1}, \mu_{2}\right)$; $e_{n}$ - единичная $(n \times n)$-матрица; $G_{1} \prec G_{2}-$ сплетение группы подстановок $G_{1}$ группой $G_{2} ; \operatorname{ord}(a)$ - порядок элемента $a \in G L_{n}(2)$; Aut $(\bar{\Gamma})$ - группа автоморфизмов графа $\bar{\Gamma}$; если $\gamma \in V_{n}, g \in G L_{n}(2), t_{\gamma}=\left|\gamma^{\langle g\rangle}\right|$ и $j \in\left\{1, \ldots, t_{\gamma}\right\}$, то

$$
\begin{aligned}
& V_{n}^{(j)}(g, \gamma)= \\
& =\left\{\gamma^{g^{i_{1}}} \oplus \ldots \oplus \gamma^{g^{i_{j}}} \mid \forall i_{1}, \ldots, i_{j} \in\left\{1, \ldots, t_{\gamma}\right\}: 0 \leq i_{1}<\ldots<i_{j} \leq t_{\gamma}-1\right\} .
\end{aligned}
$$


Всюду в работе матрицу линейного преобразования $g \in G L_{n}(2)$ в стандартном базисе $\varepsilon_{0}, \ldots, \varepsilon_{n-1}$ будем обозначать той же буквой $g$. Рассматриваемые в работе $s$-боксы биективны.

Пусть $\mu: X \times X \rightarrow \mathbb{N}_{0}$ - метрика на $X$. Натуральнозначная метрика $\rho_{\mu}: X \times X \rightarrow \mathbb{N}_{0}$, удовлетворяющая для любых элементов $\alpha, \beta, \gamma, \delta \in X$ условиям

1. если $\mu(\alpha, \beta)=\mu(\gamma, \delta)$, то $\rho_{\mu}(\alpha, \beta)=\rho_{\mu}(\gamma, \delta)$,

2. $\rho_{\mu}(\alpha, \beta) \leq \mu(\alpha, \beta)$;

называется подметрикой метрики $\mu$.

Метрика $\sigma_{\mu}: X \times X \rightarrow \mathbb{N}_{0}$ называется надметрикой метрики $\mu$, если для любых элементов $\alpha, \beta, \gamma, \delta \in X$ она удовлетворяет условиям

1. если $\sigma_{\mu}(\alpha, \beta)=\sigma_{\mu}(\gamma, \delta)$, то $\mu(\alpha, \beta)=\mu(\gamma, \delta)$,

2. $\mu(\alpha, \beta) \leq \sigma_{\mu}(\alpha, \beta)$.

Очевидно, что метрика $\mu$ является подметрикой метрики $\sigma_{\mu}$. Ряд свойств надметрик и подметрик метрики описан в работах [6-10].

\section{3. Свойства орбиталов и графов орбиталов группы $\boldsymbol{C}(\mathrm{g})$}

Напомним, что орбиталом группы подстановок $G$, действующей на множестве $X$, называется орбита группы $G$ при ее естественном действии $(\alpha, \beta)^{f}=\left(\alpha^{f}, \beta^{f}\right)$ на множестве $X^{2}$ для любого $f \in G$. Орбитал $(\alpha, \beta)^{G}$ группы $G$ называется самопарным, если $(\alpha, \beta)^{G}=(\beta, \alpha)^{G}$. Орбитал $(\alpha, \alpha)^{G}$ называется тривиальным.

Пусть $\bar{\Gamma}_{(\alpha, \beta)}(g)$ - граф произвольного нетривиального орбитала $\Gamma_{(\alpha, \beta)}(g)=(\alpha, \beta)^{C(g)}$, т. е. граф с множеством ребер $(\alpha, \beta)^{C(g)}$ и множеством вершин $\alpha^{C(g)}=V_{n}$. Поскольку группа $C(g)$ содержит все сдвиги, то ее орбиталы самопарные, а графы орбиталов неориентированные.

Очевидно, что справедливы следующие утверждения.

Лемма 1. Если g - произвольное преобразование из $G L_{n}, \alpha, \beta, \alpha^{\prime}, \beta^{\prime}-$ произвольные векторы из $V_{n}, \alpha \neq \beta$, то:

1) $\left(\alpha^{\prime}, \beta^{\prime}\right) \in(\alpha, \beta)^{C(g)}$ тогда и только тогда, когда $\alpha^{\prime} \oplus \beta^{\prime} \in(\alpha \oplus \beta)^{\langle g\rangle}$;

2) $\Gamma_{(\alpha, \beta)}(g)=\Gamma_{\left(\alpha^{\prime}, \beta^{\prime}\right)}(g)$ для любых векторов со свойством $\alpha^{\prime} \oplus \beta^{\prime} \in$ $\in(\alpha \oplus \beta)^{\langle g\rangle}$;

3) число нетривиальных орбиталов группы $C(g)$ равно $d_{g}-1$. 
Таким образом, все различные нетривиальные графы орбиталов группы $C(g)$ суть графы $\bar{\Gamma}_{\left(\overrightarrow{0}, \beta_{1}\right)}(g), \ldots, \bar{\Gamma}_{\left(\overrightarrow{0}, \beta_{d g-1}\right)}(g)$, где $\beta_{1}^{\langle g\rangle}, \ldots, \beta_{d_{g}-1}^{\langle g\rangle}-$ все различные орбиты группы $\langle g\rangle$ на $V_{n}^{\times}$. Условия изоморфизма графов орбиталов группы будут приведены в утверждениях 4 и 5.

Длина кратчайшего пути между двумя вершинами графа является расстоянием, а множество всех таких расстояний задает метрику $\mu_{\gamma, g}$, в общем случае расширенную (см., например, [7]), на множестве $V_{n}$ вершин графа $\bar{\Gamma}_{(\overrightarrow{0}, \gamma)}(g)$.

Утверждение 1. Если $g \in G L_{n}, \gamma, \alpha, \beta \in V_{n}, \gamma \neq \overrightarrow{0}, \alpha \neq \beta u t_{\gamma}=|\gamma\langle g\rangle|$, mo

$$
\begin{aligned}
& \mu_{\gamma, g}(\alpha, \beta)= \\
& =\left\{\begin{array}{l}
\infty, \text { если } \alpha \oplus \beta \notin \bigcup_{i=1}^{t_{\gamma}} V_{n}^{(i)}(g, \gamma), \\
\min \left\{i \in\left\{1, \ldots, t_{\gamma}\right\} \mid \alpha \oplus \beta \in V_{n}^{(i)}(g, \gamma)\right\}, \text { если } \alpha \oplus \beta \in \bigcup_{i=1}^{t_{\gamma}} V_{n}^{(i)}(g, \gamma) .
\end{array}\right.
\end{aligned}
$$

Доказательство при $\mu_{\gamma, g}(\alpha, \beta) \neq \infty$ проводится индукцией по расстоянию $\mu_{\gamma, g}(\alpha, \beta)$, и мы его опускаем.

Следствие 1. Если $g \in G L_{n} u \gamma \in V_{n}^{\times}$, то:

1) степень каждой вершины графа $\bar{\Gamma}_{(\overrightarrow{0}, \gamma)}(g)$ равна $\left|\gamma^{\langle g\rangle}\right|$;

2) граф $\bar{\Gamma}_{(\overrightarrow{0}, \gamma)}(g)$ связен тогда и только тогда, когда $\bigcup_{i=1}^{t_{\gamma}} V_{n}^{(i)}(g, \gamma)=V_{n}$;

3) граф $\bar{\Gamma}_{(\overrightarrow{0}, \gamma)}$ связен тогда и только тогда, когда $\langle\gamma\langle g\rangle\rangle=V_{n}$;

4) если граф $\Gamma_{(\overrightarrow{0}, \gamma)}(g)$ не связен, $\left\{\theta_{1}, \ldots, \theta_{r}\right\}-$ максимальное линейно независимое подмножество мощности $r$ множества $\gamma^{\langle g\rangle}, \bar{\Theta}=\left\langle\theta_{1}, \ldots, \theta_{r}\right\rangle$,

$$
V_{n} / \bar{\Theta}=\left\{\alpha_{0} \oplus \bar{\Theta}, \alpha_{1} \oplus \bar{\Theta}, \ldots, \alpha_{2^{n-r}-1} \oplus \bar{\Theta}\right\}, \quad \alpha_{0}=\overrightarrow{0},
$$

то граф $\bar{\Gamma}_{(\overrightarrow{0}, \gamma)}(g)$ имеет изоморфные компоненты связности $\bar{\Gamma}_{(\overrightarrow{0}, \gamma)}^{(i)}(g)$ с множеством вершин $\alpha_{i} \oplus \bar{\Theta}, i=0, \ldots, 2^{n-r}-1$;

5) диаметр связного графа $\bar{\Gamma}_{(\overrightarrow{0}, \gamma)}(g)$ не превосходит $n$, диаметр этого графа равен п тогда и только тогда, когда $\gamma^{\langle g\rangle}-$ базис $V_{n}$.

Доказательство. Пп. 1)-2) следуют из утверждения 1. 
3) Согласно п. 2) достаточно показать эквивалентность равенств $\bigcup_{i=1}^{t_{\gamma}} V_{n}^{(i)}(g, \gamma)=V_{n}$ и $\left\langle\gamma^{\langle g\rangle}\right\rangle=V_{n}$. Последнее следует из равенства

$$
\left\{\gamma c_{0} \oplus \gamma^{g} c_{1} \oplus \ldots \oplus \gamma^{g^{t_{\gamma}-1}} c_{t_{\gamma}-1} \mid\left(c_{t_{\gamma}-1}, \ldots, c_{0}\right) \in V_{n}\right\}=\bigcup_{i=1}^{t_{\gamma}} V_{n}^{(i)}(g, \gamma)
$$

4) Очевидно, что $r=\operatorname{deg}\left(m_{\gamma, g}(x)\right)$. Аналогично утверждению 1 показывается, что вершины $\beta, \beta^{\prime}$ принадлежат одной компоненте связности графа $\bar{\Gamma}_{(\overrightarrow{0}, \gamma)}(g)$ тогда и только тогда, когда $\beta \oplus \beta^{\prime} \in \bar{\Theta}$. Следовательно, $\alpha_{i} \oplus \bar{\Theta} \in$ $\in V_{n} / \bar{\Theta}-$ множество вершин компоненты связности $\bar{\Gamma}_{(\overrightarrow{0}, \gamma)}^{(i)}(g)$ графа $\bar{\Gamma}_{(\overrightarrow{0}, \gamma)}(g)$, $i=0, \ldots, 2^{n-r}-1$.

Зафиксируем две произвольные различные компоненты связности $\bar{\Gamma}_{(\overrightarrow{0}, \gamma)}^{(i)}(g), \bar{\Gamma}_{(\overrightarrow{0}, \gamma)}^{(j)}(g)$ и зададим отображение $\psi_{i j}: \alpha_{i} \oplus \theta \mapsto \alpha_{j} \oplus \theta, \theta \in \bar{\Theta}$. Согласно утверждению 1 вершины $\alpha_{i} \oplus \lambda, \alpha_{i} \oplus \lambda^{\prime}$ смежны в графе $\bar{\Gamma}_{(\overrightarrow{0}, \gamma)}^{(i)}(g)$ тогда и только тогда, когда $\lambda \oplus \lambda^{\prime} \in \gamma^{\langle g\rangle}$. Но вершины $\psi_{i j}\left(\alpha_{i} \oplus \lambda\right)=$ $\alpha_{j} \oplus \lambda, \psi_{i j}\left(\alpha_{i} \oplus \lambda^{\prime}\right)=\alpha_{j} \oplus \lambda^{\prime}$ в графе $\bar{\Gamma}_{(\overrightarrow{0}, \gamma)}^{(j)}(g)$ являются смежными тогда и только тогда, когда $\lambda \oplus \lambda^{\prime} \in \gamma^{\langle g\rangle}$. Значит, $\psi_{i j}-$ изоморфизм и граф $\bar{\Gamma}_{(\overrightarrow{0}, \gamma)}(g)$ имеет $2^{n-r}$ изоморфных компонент связности.

5) Согласно п. 3 и в силу связности графа $\bar{\Gamma}_{(\overrightarrow{0}, \gamma)}(g)$ имеем включение $\left\langle\gamma^{\langle g\rangle}\right\rangle \subseteq V_{n}$. Пусть $\left\{\theta_{1}, \ldots, \theta_{n}\right\} \subseteq \gamma^{\langle g\rangle}$ для некоторого базиса $\theta_{1}, \ldots, \theta_{n}$ пространства $V_{n}$.

Если $\left\{\theta_{1}, \ldots, \theta_{n}\right\}=\gamma^{\langle g\rangle}$, то расстояние между вершинами графа $\bar{\Gamma}_{(\overrightarrow{0}, \gamma)}(g)$ равно $n$ по утверждению 1.

Если $\left\{\theta_{1}, \ldots, \theta_{n}\right\} \subset \gamma^{\langle g\rangle}$, то $V_{n}^{(n)}(g, \gamma) \subseteq \bigcup_{j=1}^{n-1} V_{n}^{(j)}(g, \gamma)$. Значит, $\mu_{\gamma, g}(\alpha, \beta) \leq n-1$ для любых векторов $\alpha, \beta \in V_{n}$. Следствие доказано.

Существует тесная связь между строением характеристического многочлена $u_{g}(x)$, примитивностью (или 2-транзитивностью) и связностью графов орбиталов группы $C(g)$.

Утверждение 2. 1. Группа $C(g)$ примитивна (2-транзитивна) тогда и только тогда, когда многочлен $u_{g}(x)$ неприводим (примитивен).

2. Граф $\bar{\Gamma}_{(\overrightarrow{0}, \gamma)}(g)$ связен тогда и только тогда, когда многочлен $u_{g}(x)$ неприводим.

Доказательство. П. 1 очевиден. 
2. По п. 1 примитивность группы $C(g)$ равносильна неприводимости многочлена $u_{g}(x)$. Но группа примитивна тогда и только тогда, когда графы всех ее нетривиальных орбиталов связны (см., например, [1]). Следовательно, связность графа $\bar{\Gamma}_{(\overrightarrow{0}, \gamma)}(g)$ для всех $\gamma \in V_{n}^{\times}$равносильна неприводимости многочлена $u_{g}(x)$. Утверждение доказано.

Приведем критерий связности графа $\bar{\Gamma}_{(\overrightarrow{0}, \gamma)}(g)$ на языке минимального многочлена $m_{\gamma, g}(x)$ и покажем, что примитивность группы $C(g)$ накладывает сильные ограничения на ее графы орбиталов, в частности, влечет их изоморфизм. В этом случае XL-слой имеет меньшую структурированность.

Следствие 2. 1. Граф $\bar{\Gamma}_{(\overrightarrow{0}, \gamma)}(g)$ связен тогда и только тогда, когда $m_{\gamma, g}(x)=u_{g}(x)$.

2. Если группа $C(g)$ примитивна, то графы всех ее нетривиальных орбиталов изоморфны.

Доказательство. 1. Пусть граф $\bar{\Gamma}_{(\overrightarrow{0}, \gamma)}(g)$ связен. Согласно утверждению 19 [2] существует такое $k \in\{1, \ldots, n\}$, что система векторов $\gamma, \gamma^{g}, \ldots, \gamma^{g^{k-1}}$ линейно независима, а вектор $\gamma^{g^{k}}$ линейно выражается через эту систему и

$$
m_{\gamma, g}(x)=x^{k} \oplus c_{k-1} x^{k-1} \oplus \ldots \oplus c_{1} x \oplus c_{0},
$$

где

$$
\gamma^{g^{k}}=c_{k-1} \gamma^{g^{k-1}} \oplus \ldots \oplus c_{1} \gamma^{g} \oplus \gamma c_{0}, \quad c_{0}, \ldots, c_{k-1} \in\{0,1\} .
$$

Поскольку граф $\bar{\Gamma}_{(\overrightarrow{0}, \gamma)}(g)$ связен и $m_{\gamma, g}(x) \mid u_{g}(x)$, то $k=n$ и $m_{\gamma, g}(x)=u_{g}(x)$.

Обратно, пусть $m_{\gamma, g}(x)=u_{g}(x)$. Покажем, что граф $\bar{\Gamma}_{(\overrightarrow{0}, \gamma)}(g)$ связен. Так как $m_{\gamma, g}(x)=u_{g}(x)$, то $\operatorname{deg}\left(m_{\gamma, g}(x)\right)=n$. Из утверждения 19 [2] получаем, что система $\gamma, \gamma^{g}, \ldots, \gamma^{g^{n-1}}$ линейно независима. Теперь из следствия 1 получаем, что граф $\bar{\Gamma}_{(\overrightarrow{0}, \gamma)}(g)$ связен.

2. Пусть $\bar{\Gamma}_{\left(\overrightarrow{0}, \gamma_{1}\right)}(g), \bar{\Gamma}_{\left(\overrightarrow{0}, \gamma_{2}\right)}(g)$ - два произвольных различных графа, группа $C(g)$ примитивна и $\gamma_{\varepsilon i}=\gamma_{\varepsilon}^{g^{i}}, i=0, \ldots, n-1, \varepsilon=1,2$. Тогда $m_{\gamma_{1}, g}(x)=m_{\gamma_{2}, g}(x)=u_{g}(x)$ и $\gamma_{10}, \ldots, \gamma_{1 n-1}$ и $\gamma_{20}, \ldots, \gamma_{2 n-1}-$ базисы пространства $V_{n}$. Существует такое линейное преобразование $h \in G L_{n}$, что $\gamma_{1 i}^{h}=\gamma_{2 i}, i=0, \ldots, n-1$. Из равенств

$$
\left(\gamma_{1}^{g^{n}}\right)^{h}=\left(c_{n-1} \gamma_{1}^{g^{n-1}} \oplus \ldots \oplus c_{1} \gamma_{1}^{g} \oplus \gamma_{1} c_{0}\right)^{h}=c_{n-1} \gamma_{2}^{g^{n-1}} \oplus \ldots \oplus c_{1} \gamma_{2}^{g} \oplus \gamma_{2} c_{0}=\gamma_{2}^{g^{n}}
$$

следует, что $\gamma_{1 n}^{h}=\gamma_{2 n}$ и $\gamma_{1 i}^{h}=\gamma_{2 i}, \gamma_{1 i}^{g h}=\gamma_{2 i}, i=0, \ldots, n-1$. Поэтому $\gamma_{1 i}^{g h g^{-1} h^{-1}}=\gamma_{2 i}$ для $i=0, \ldots, n-1$ и $g h=h g, \gamma_{1}^{g j h}=\gamma_{2}^{g j}$ для $j=1, \ldots,\left|\gamma_{1}^{\langle g\rangle}\right|$. 
Значит, $\left(\gamma_{1}^{\langle g\rangle}\right)^{h}=\gamma_{2}^{\langle g\rangle}$ и графы $\Gamma_{\left(\overrightarrow{0}, \gamma_{1}\right)}(g), \Gamma_{\left(\overrightarrow{0}, \gamma_{2}\right)}(g)$ изоморфны. Следствие доказано.

Опишем зависимость свойств графов $\bar{\Gamma}_{\left(\overrightarrow{0}, \gamma_{1}\right)}(g), \ldots, \bar{\Gamma}_{\left(\overrightarrow{0}, \gamma_{l}\right)}(g)$ и метрик $\mu_{\gamma_{1}, g}, \ldots, \mu_{\gamma_{l}, g}$ от свойств характеристического многочлена $u_{g}(x)$.

Если многочлен $u_{g}(x)$ неприводим, то из утверждения 2 и следствия 2 получаем равенство длин орбит группы $\langle g\rangle$.

Если $u_{g}(x)=f_{1}^{r_{1}}(x) \ldots f_{b}^{r_{b}}(x)$, где $f_{i}(x)$ - различные неприводимые многочлены, $\operatorname{deg}\left(f_{i}(x)\right)=n_{i}>0, i=1, \ldots, b$, то матрица $g$ подобна клеточнодиагональной матрице $g^{\prime}=\operatorname{diag}\left(g_{1}^{\prime}, \ldots, g_{b}^{\prime}\right), u_{g_{i}^{\prime}}(x)=f_{i}^{r_{i}}(x), i=1, \ldots, b$. Матрица $g$ определена однозначно с точностью до подобия и порядка следования клеток. Можем перейти к рассмотрению подобной группы $C\left(g^{\prime}\right)(\approx C(g))$.

Утверждение 3. 1. Если матрица g неразложима над $G F(2)$, то хотя бы один из графов орбиталов группы $C(g)$ связен.

2. Если матрица g разложима над $G F(2), b>1, u_{g}(x)=f_{1}(x) \ldots f_{b}(x)$, $f_{1}(x), \ldots, f_{b}(x)$ - попарно различные неприводимые многочлены ненулевой степени, $\operatorname{deg}\left(f_{i}(x)\right)=n_{i}, \gamma=\left(\gamma^{(1)}, \ldots, \gamma^{(b)}\right) \in V_{n_{1}}^{\times} \ldots \times V_{n_{b}}$, то связность графа $\bar{\Gamma}_{(\overrightarrow{0}, \gamma)}(g)$ равносильна условию $\gamma^{(i)} \neq 0, i=1, \ldots, b$.

Доказательство. 1. Пусть матрица $g$ неразложима над $G F(2)$. Тогда $u_{g}(x)=m_{g}(x)=f(x)^{k}, f(x)-$ неприводимый многочлен. Согласно следствию 2 граф $\bar{\Gamma}_{(\overrightarrow{0}, \gamma)}(g)$ связен тогда и только тогда, когда $m_{\gamma, g}(x)=u_{g}(x)$.

Так как $m_{g}(x)=f(x)^{k}=\operatorname{HOK}\left(m_{\delta_{1}, g}(x), \ldots, m_{\delta_{n}, g}(x)\right)$, где векторы $\delta_{1}, \ldots, \delta_{n}$ линейно независимы, то найдется такой вектор $\delta_{j}$, что $m_{\delta_{j}, g}(x)=$ $=f(x)^{k}$ и граф $\bar{\Gamma}_{\left(\overrightarrow{0}, \delta_{j}\right)}(g)$ связен.

2. Пусть $\theta=\left(\theta^{(1)}, \ldots, \theta^{(b)}\right), \theta^{(i)} \in V_{n_{i}}^{\times}, i=1, \ldots, b$. Из утверждения 2 следует, что $m_{\theta^{(i)}, g}(x)=m_{\theta^{(i)}, g_{i}^{\prime}}(x)=f_{i}(x), i=1, \ldots, b$. Тогда $m_{\theta, g}(x)=f_{1}(x) \times$ $\times \ldots \cdot f_{b}(x)=u_{g}(x)$. По следствию 2 граф $\bar{\Gamma}_{(\overrightarrow{0}, \theta)}(g)$ связен.

Пусть $\gamma^{(i)}=0$ для некоторого $i \in\{1, \ldots, b\}$ и $m_{g, \gamma}^{\prime}(x)=$ $=\left(\prod_{j=1}^{b} f_{j}(x)\right) / f_{i}(x)$. Тогда $m_{g, \gamma}^{\prime}(\gamma)=(0, \ldots, 0)$, т. е. $m_{\gamma, g}^{\prime}(x) \neq u_{g}(x)$. Согласно следствию 2 граф $\bar{\Gamma}_{(\overrightarrow{0}, \gamma)}(g)$ не связен. Утверждение доказано.

Приведем пример такой разложимой над $G F(2)$ матрицы $g$, что $u_{g}(x)=$ $=f(x)^{k}$ и все графы орбиталов группы $C(g)$ не связны.

Пусть $n=2^{t+1}$ для некоторого $t \in \mathbb{N}$ и преобразованию $g$ соответствует перестановка координат $\bar{g}$ векторов пространства $V_{n}$, т. е. действует как $\left(\alpha_{n-1}, \ldots, \alpha_{0}\right)^{g}=\left(\alpha_{(n-1)^{\bar{g}}}, \ldots, \alpha_{0 \bar{g}}\right)$, где $\bar{g}=\left(0, \ldots, 2^{t}-1\right)\left(2^{t}, \ldots, 2^{t+1}-1\right)$. 
Тогда $g$ - разложимая матрица с характеристическим многочленом $u_{g}(x)=$ $=(x \oplus 1)^{n}$, а минимальный многочлен любого вектора делит $x^{2^{n-1}} \oplus 1$. По следствию 2 графы всех орбиталов группы $C(g)$ не связны. Подобные преобразования могут возникать в XSL-шифрсистемах.

Приведем условие изоморфизма графов $\bar{\Gamma}_{\left(\overrightarrow{0}, \gamma_{1}\right)}(g), \bar{\Gamma}_{\left(\overrightarrow{0}, \gamma_{2}\right)}(g)$.

Утверждение 4. Два различных графа $\bar{\Gamma}_{\left(\overrightarrow{0}, \gamma_{1}\right)}(g), \bar{\Gamma}_{\left(\overrightarrow{0}, \gamma_{2}\right)}(g)$ изоморфны тогда и только тогда, когда

$$
\psi(\alpha) \oplus \psi\left(\alpha \oplus \gamma_{1}^{\langle g\rangle}\right)=\gamma_{2}^{\langle g\rangle}
$$

для некоторого преобразования $\psi \in S\left(V_{n}\right)$ и каждого вектора $\alpha \in V_{n}$.

Доказательство. Изоморфизм графов $\bar{\Gamma}_{\left(\overrightarrow{0}, \gamma_{1}\right)}(g)$ и $\bar{\Gamma}_{\left(\overrightarrow{0}, \gamma_{2}\right)}(g)$ равносилен существованию такого биективного отображения $\psi: V_{n} \stackrel{\rightarrow}{\rightarrow} V_{n}$, что $\psi(\alpha) \oplus$ $\oplus \psi(\beta) \in \gamma_{2}^{\langle g\rangle}$, если $\alpha, \beta \in V_{n}$ и $\alpha \oplus \beta \in \gamma_{1}^{\langle g\rangle}$. Поэтому из равенства $\psi(\alpha) \oplus$ $\oplus \psi\left(\alpha \oplus \gamma_{1}^{\langle g\rangle}\right)=\gamma_{2}^{\langle g\rangle}$ получаем изоморфизм графов $\bar{\Gamma}_{\left(\overrightarrow{0}, \gamma_{1}\right)}(g), \bar{\Gamma}_{\left(\overrightarrow{0}, \gamma_{2}\right)}(g)$.

Если графы изоморфны, то $\psi(\alpha) \oplus \psi\left(\alpha \oplus \gamma_{1}^{\langle g\rangle}\right) \subseteq \gamma_{2}^{\langle g\rangle}$ для любого вектора $\alpha \in V_{n}$. Согласно п. 1 следствия 1 степень каждой вершины графа $\bar{\Gamma}_{(\overrightarrow{0}, \gamma)}(g)$ равна $\left|\gamma^{\langle g\rangle}\right|$. Значит, $\left|\gamma_{1}^{\langle g\rangle}\right|=\left|\gamma_{2}^{\langle g\rangle}\right|$, и выполняется равенство $\psi(\alpha) \oplus$ $\psi\left(\alpha \oplus \gamma_{1}^{\langle g\rangle}\right)=\gamma_{2}^{\langle g\rangle}$. Утверждение доказано.

Утверждение 5. Пусть $n \geq 2, \gamma_{1}, \gamma_{2} \in V_{n}^{\times}$. Условия $\bar{\Gamma}_{\left(\overrightarrow{0}, \gamma_{1}\right)}(g) \cong$ $\cong \bar{\Gamma}_{\left(\overrightarrow{0}, \gamma_{2}\right)}(g) u m_{\gamma_{1}, g}(x)=m_{\gamma_{2}, g}(x)$ равносильны.

Доказательство. Из утверждения 4 следует, что существование изоморфизма $\bar{\Gamma}_{\left(\overrightarrow{0}, \gamma_{1}\right)}(g) \cong \bar{\Gamma}_{\left(\overrightarrow{0}, \gamma_{2}\right)}(g)$ равносильно существованию преобразования $\psi \in S\left(V_{n}\right)$, удовлетворяющего равенству (1). Покажем, что преобразование $\psi$ можно взять линейным.

Отметим, что для линейного преобразования $\psi$ равенство (1) равносильно равенству $\psi\left(\gamma_{1}^{\langle g\rangle}\right)=\gamma_{2}^{\langle g\rangle}$.

Пусть

$$
m_{\gamma_{1}}(x)=m_{\gamma_{2}}(x)=x^{d} \oplus c_{d-1} x^{d-1} \oplus \ldots \oplus c_{1} x \oplus c_{0} .
$$

Положим $\Lambda(\gamma)=\left\{\gamma^{g^{i}} \mid i \in\{0, \ldots, d-1\}\right\}$ для $\gamma \in V_{n}$. Так как $\operatorname{deg}\left(m_{\gamma_{i}}(x)\right)=d$, то векторы из множества $\Lambda\left(\gamma_{j}\right)$ линейно независимы, $j=1,2$. Поэтому существует такое линейное преобразование $u \in G L_{n}(2)$, что $\gamma_{2}^{g^{i}}=\gamma_{1}^{g^{i} u}, i=0, \ldots, d-1$. 
Аналогично доказательству следствия 2 показываем, что $\gamma_{2}^{g^{i}}=\gamma_{1}^{g^{i} u}$, $i=d, \ldots,\left|\gamma_{1}^{\langle g\rangle}\right|$. Значит, $\left(\gamma_{1}^{\langle g\rangle}\right)^{u}=\gamma_{2}^{\langle g\rangle}$. Утверждение доказано.

\section{4. Некоторые классы графов орбиталов группы $C(g)$}

В алгебраической теории графов наибольший интерес представляют вершинно-транзитивные, реберно-транзитивные, дистанционно-транзитивные и дистанционно-регулярные графы [3].

Напомним, что граф $\bar{\Gamma}=(X, Y)$ с множеством вершин $X$ и множеством ребер $Y$ называется дистанщионно-транзитивным, если для любых вершин $\alpha, \alpha^{\prime}, \beta, \beta^{\prime} \in X$, для которых $\mu\left(\alpha, \alpha^{\prime}\right)=\mu\left(\beta, \beta^{\prime}\right)$, существует элемент $g \in \operatorname{Aut}(\bar{\Gamma})$, удовлетворяющий равенствам $\beta=\alpha^{g}, \beta^{\prime}=\alpha^{g}$, где $\mu\left(\alpha, \alpha^{\prime}\right)-$ расстояние между вершинами $\alpha, \alpha^{\prime}$ в графе $\bar{\Gamma}$. Граф $\bar{\Gamma}=(X, Y)$ называется вериинно-транзитивным (реберно-транзитивным), если группа Aut $(\bar{\Gamma})$ действует на множестве $X$ (соответственно $Y$ ) транзитивно.

Имеется ряд эквивалентных определений дистанционно-регулярных графов. Нам понадобится следующее определение.

Конечный граф $\bar{\Gamma}$ называется дистанционно-регулярным, если для любых $i, j \in \mathbb{N}_{0}$ и любых $\alpha^{\prime}, \beta^{\prime} \in X$, для которых $\mu\left(\alpha^{\prime}, \beta^{\prime}\right)=j$, число $p_{1, i}^{(j)}$ таких вершин $\delta \in X$, что $\mu\left(\alpha^{\prime}, \delta\right)=1, \mu\left(\delta, \beta^{\prime}\right)=i$, зависит от $i, j$, но не зависит от вершин $\alpha^{\prime}, \beta^{\prime}$.

Дистанционная регулярность графа тесно связана с понятием $P$-полиномиальной схемы отношений (см., например, [8]) - активно изучаемым объектом в алгебраической комбинаторике. Так, граф является дистанционно-регулярным, если степени его матрицы смежности порождают схему отношений. Дистанционно-транзитивный граф дистанционнорегулярен. В [4] описаны графы орбиталов надгрупп группы Хемминга, являющиеся дистанционно-транзитивными или дистанционно-регулярными.

Графом Хемминга на $V_{n}$ называется граф с множеством вершин $V_{n}$ и множеством ребер $\left\{(\alpha, \beta) \in V_{n}^{2} \mid \chi_{n}(\alpha, \beta)=1\right\}$. Очевидно, что если граф изоморфен графу Хемминга, то его метрика изоморфна метрике Хемминга.

Лемма 2. Пусть $n \geq 2, i \in\left\{1, \ldots, d_{g}-1\right\}, \bar{\Gamma}_{\left(\overrightarrow{0}, \gamma_{i}\right)}(g)-$ связный граф с диаметром, не меньшим двух. Тогда

1) граф $\bar{\Gamma}_{\left(\overrightarrow{0}, \gamma_{i}\right)}(g)$ реберно-транзитивныц

2) если $\gamma_{i}^{\langle g\rangle}$ есть базис пространства $V_{n}$, то граф $\bar{\Gamma}_{\left(\overrightarrow{0}, \gamma_{i}\right)}(g)$ дистанционно-транзитивный и Aut $\bar{\Gamma}_{\left(\overrightarrow{0}, \gamma_{i}\right)}(g) \approx S_{2} \uparrow S_{n}$. 
Доказательство. Пусть $V_{n}^{(c)}(g, \gamma, \alpha)=\left\{\theta \in V_{n} \mid \mu_{\gamma, g}(\alpha, \theta)=c\right\}$.

1) Если $\left(\alpha, \alpha^{\prime}\right)$ и $\left(\beta, \beta^{\prime}\right)-$ peбра графа $\bar{\Gamma}_{\left(\overrightarrow{0}, \gamma_{i}\right)}(g)$, то $\alpha^{\prime}=\alpha \oplus \gamma_{i}^{g^{c}}, \beta^{\prime}=$ $=\beta \oplus \gamma_{i}^{g^{r}}$ для некоторых $c, r \in\left\{0, \ldots,\left|\gamma_{i}^{\langle g\rangle}\right|-1\right\}$ и выполняются равенства

$$
\begin{aligned}
& \left(\alpha, \alpha \oplus \gamma_{i}^{g^{c}}\right)^{g^{r-c}}=\left(\alpha^{g^{r-c}}, \alpha^{g^{r-c}} \oplus \gamma_{i}^{g^{r}}\right), \\
& \left(\alpha^{g^{r-c}}, \alpha^{g^{r-c}} \oplus \gamma_{i}^{g^{r}}\right)^{h_{\lambda}}=\left(\beta, \beta \oplus \gamma_{i}^{g^{r}}\right),
\end{aligned}
$$

где $\lambda=\alpha^{g^{r-c}} \oplus \beta$. Таким образом, $\left(\alpha, \alpha^{\prime}\right)^{b}=\left(\beta, \beta^{\prime}\right)$ для некоторого $b=$ $=g^{r-c} h_{\gamma} \in \operatorname{Aut}\left(\bar{\Gamma}_{\left(\overrightarrow{0}, \gamma_{i}\right)}(g)\right)$.

2) Если $\gamma_{i}^{\langle g\rangle}$ является базисом $V_{n}$, то из утверждений 1 и 4 следует, что граф $\bar{\Gamma}_{\left(\overrightarrow{0}, \gamma_{i}\right)}(g)$ изоморфен графу Хемминга с метрикой $\chi_{n}$. Граф Хемминга является дистанционно-транзитивным, а $S_{2} \uparrow S_{n}$ - его группа автоморфизмов. Лемма доказана.

Отметим, что если множество $\gamma_{i}^{\langle g\rangle}-$ базис $V_{n}$, то $\bar{\Gamma}_{\left(\overrightarrow{0}, \gamma_{i}\right)}(g)-$ дистанционно-регулярный граф, изоморфный графу Хемминга.

Теорема 1. Пусть $n \geq 2$ и граф $\bar{\Gamma}_{(\overrightarrow{0}, \gamma)}(g)$ связен.

1. Граф $\bar{\Gamma}_{(\overrightarrow{0}, \gamma)}(g)$ изоморфен графу Хемминга с метрикой $\chi_{n}$ тогда и только тогда, когда $m_{\gamma, g}(x)=x^{n} \oplus 1$.

2. Пусть $m_{\gamma, g}(x)=x^{r} \oplus 1$ для некоторого $r \in\{1, \ldots, n\}$. Граф $\bar{\Gamma}_{(\overrightarrow{0}, \gamma)}(g)$ дистанционно-регулярен тогда и только тогда, когда $r=n$.

3. Пусть $m_{\gamma, g}(x)=x^{r(q-1)} \oplus x^{r(q-2)} \oplus \ldots \oplus x^{r} \oplus 1=\frac{\left(x^{r}\right)^{q}-1}{x^{r}-1}, r q=m=$ $=\left|\gamma^{\langle g\rangle}\right|$. Граф $\bar{\Gamma}_{(\overrightarrow{0}, \gamma)}(g)$ дистанщионно-регулярен тогда и только тогда, когда $r=1$ или $r \geq 2$ и $q=3$.

Доказательство. 1. Рассмотрим такое преобразование $a \in G L_{n}$, что

$$
\left(\alpha_{n-1}, \ldots, \alpha_{0}\right)^{a}=\left(\alpha_{1}, \ldots, \alpha_{n-1}, \alpha_{0}\right)
$$

для любого вектора $\left(\alpha_{n-1}, \ldots, \alpha_{0}\right) \in V_{n}$. Тогда $\varepsilon_{n-1}^{\langle a\rangle}=\left\langle\varepsilon_{0}, \ldots, \varepsilon_{n-1}\right\rangle=V_{n}$. Нетрудно убедиться, что $\bar{\Gamma}_{\left(\overrightarrow{0}, \varepsilon_{n-1}\right)}(a)$ есть граф Хемминга с метрикой $\chi_{n}$ и $m_{\varepsilon_{n-1}, a}(x)=x^{n} \oplus 1$. Из утверждения 5 получаем, что условие $\bar{\Gamma}_{(\overrightarrow{0}, \gamma)}(g) \cong$ $\cong \bar{\Gamma}_{\left(\overrightarrow{0}, \varepsilon_{n-1}\right)}(a)$ равносильно равенству $m_{\gamma, g}(x)=m_{\varepsilon_{n-1}, a}(x)$, т. е. $m_{\gamma, g}(x)=$ $=x^{n} \oplus 1$.

2. Пусть $\gamma^{\langle g\rangle}=\left\{\delta_{0}, \ldots, \delta_{m-1}\right\}$ и граф $\bar{\Gamma}_{(\overrightarrow{0}, \gamma)}(g)$ дистанционно-регулярный. Так как $m_{\gamma, g}(x)=x^{r} \oplus 1$, то $r=m$. Из связности графа $\bar{\Gamma}_{(\overrightarrow{0}, \gamma)}(g)$ следует, 
что $\left\langle\gamma^{\langle g\rangle}\right\rangle=V_{n}$ и $m=n$. По лемме 4.1 граф $\bar{\Gamma}_{(\overrightarrow{0}, \gamma)}(g)$ изоморфен графу Хемминга с метрикой $\chi_{n}$.

Доказательство в обратную сторону следует из п. 1.

3. Из связности графа и следствия 2 получаем, что $n=r(q-1)$. Пусть $\Theta_{t}=\left\{\theta_{1}^{(1)}, \ldots, \theta_{t}^{(1)}, \ldots, \theta_{1}^{(r)}, \ldots, \theta_{t}^{(r)}\right\} \subseteq V_{n}$ для $t \in\{2, \ldots, q\}$, причем $\Theta_{q-1}-$ произвольный базис $V_{n}, \Theta=\Theta_{q}$ и $\theta_{q}^{(1)}=\theta_{1}^{(1)} \oplus \ldots \oplus \theta_{q-1}^{(1)}, \ldots, \theta_{q}^{(r)}=\theta_{1}^{(r)} \oplus$ $\oplus \ldots \oplus \theta_{q-1}^{(r)}$.

Так как $\operatorname{deg} m_{\gamma, g}(x)=(q-1) r=n$, то векторы $\gamma, \gamma^{g}, \ldots, \gamma^{(q-1) r-1}$ линейно независимы и равенства $\varphi\left(\gamma^{g^{(j-1) r+i-1}}\right)=\theta_{j}^{(i)}, i=1, \ldots, r, j=$ $=1, \ldots, q-1$, задают линейное отображение $\varphi: V_{n} \rightarrow V_{n}$. Так как $m_{\gamma, g}(x)=$ $=u_{g}(x)$, то $\varphi\left(\gamma^{g^{(q-1) r+i-1}}\right)=\theta_{q}^{(i)}, i=1, \ldots, r$.

Рассмотрим граф $\bar{\Gamma}_{\Theta}$ с множеством вершин $V_{n}$ и множеством ребер $\left\{(\alpha, \beta) \in V_{n}^{2} \mid \alpha \oplus \beta \in \Theta\right\}$. Пусть $\mu_{\Theta}(\alpha, \beta)-$ расстояние между вершинами $\alpha, \beta$ графа $\bar{\Gamma}_{\Theta}, W_{n}^{(c)}\left(\bar{\Gamma}_{\Theta}, \alpha\right)=\left\{\theta \in V_{n} \mid \mu_{\Theta}(\alpha, \theta)=c\right\}$ и

$$
\begin{gathered}
W_{n, i}^{(j)}=\left\{\theta_{b_{1}}^{(j)} \oplus \ldots \oplus \theta_{b_{i}}^{(j)} \mid 1 \leq b_{1}<\ldots<b_{i} \leq q\right\}, \quad W_{n, i_{1}, i_{2}}^{(j)}=W_{n, i_{1}}^{(j)} \cup W_{n, i_{2}}^{(j)}, \\
\Theta^{(j)}=\left\{\theta_{1}^{(j)}, \ldots, \theta_{q-1}^{(j)}\right\}, \quad i, i_{1}, i_{2} \in\{1, \ldots, q-1\}, \quad j=1, \ldots, r .
\end{gathered}
$$

Рассмотрим граф орбитала $\bar{\Gamma}_{1 \cup(q-1)}$ надгруппы группы Джевонса (в обозначениях работы [6]), т. е. граф, являющийся объединением некоторых графов орбиталов группы Джевонса и имеющий множество вершин $V_{q-1}$ и множество ребер $\left\{(\alpha, \beta) \in V_{q-1}^{2} \mid \alpha \oplus \beta \in \Omega\right\}$, где $\Omega=\left\{\varepsilon_{0}, \ldots, \varepsilon_{q-2}, \varepsilon_{0} \oplus \ldots \oplus \varepsilon_{q-2}\right\}$. Такой граф возник в [5] как граф орбитала надгруппы группы $S_{2} \uparrow S_{n}$. Его дистанционная транзитивность доказана в [6].

Отметим, что графы $\bar{\Gamma}_{\Theta(j)}$ с множеством вершин $\left\langle\Theta^{(j)}\right\rangle$ и множеством ребер $\left\{(\alpha, \beta) \in\left\langle\Theta^{(j)}\right\rangle^{2} \mid \alpha \oplus \beta \in \Theta^{(j)}\right\}$ дистанционно-регулярны, так как изоморфны дистанционно-регулярному графу $\bar{\Gamma}_{1 \cup(q-1)}$ с множеством вершин $V_{q-1}, j \in\{1, \ldots, r\}$. При этом справедливо равенство $\bar{\Gamma}_{1 \cup(q-1)}=\bar{\Gamma}_{\Omega}$. Кроме того,

$$
\begin{aligned}
& W_{n}^{(j)}\left(\bar{\Gamma}_{1 \cup(q-1)}, \overrightarrow{0}\right)=\left\{\varepsilon_{r_{1}} \oplus \ldots \oplus \varepsilon_{r_{j}} \mid 1 \leq r_{1}<\ldots<r_{j} \leq q-1\right\} \cup \\
& \cup\left\{\varepsilon_{r_{1}} \oplus \ldots \oplus \varepsilon_{r_{q-j}} \mid 1 \leq r_{1}<\ldots<r_{q-j} \leq q-1\right\}, \quad j=1, \ldots,\lceil(q-1) / 2\rceil .
\end{aligned}
$$

Из задания отображения $\varphi$ следует эквивалентность соотношений $\alpha \oplus$ $\oplus \beta \in \gamma^{\langle g\rangle}$ и $(\varphi(\alpha), \varphi(\beta)) \in \Theta_{q}$. Следовательно, $\bar{\Gamma}_{(\overrightarrow{0}, \gamma)}(g) \cong \bar{\Gamma}_{\Theta}$.

Дальше нам понадобится следующая лемма. 
Лемма 3. Пусть $\Lambda$ - непустое подмножество из $V_{n}, \bar{\Gamma}_{\Lambda}-$ граф с множеством вершин $V_{n}$, множеством ребер $\left\{(\alpha, \beta) \in V_{n}^{2} \mid \alpha \oplus \beta \in \Lambda\right\}$ и расстоянием $\mu_{\Lambda}(\alpha, \beta)$ между вершинами,

$$
W_{n}^{(i)}\left(\bar{\Gamma}_{\Lambda}, \alpha\right)=\left\{\theta \in V_{n} \mid \mu_{\Lambda}(\alpha, \theta)=i\right\}, \quad i=1,2, \ldots,
$$

и $p_{i j}^{(t)}\left(\theta_{1}\right)$ - число таких векторов $\theta_{2} \in V_{n}^{(i)}\left(\bar{\Gamma}_{\Lambda}, \overrightarrow{0}\right)$, что $\theta_{1} \oplus \theta_{2} \in V_{n}^{(j)}\left(\bar{\Gamma}_{\Lambda}, \overrightarrow{0}\right)$, где $\theta_{1} \in V_{n}^{(t)}\left(\bar{\Gamma}_{\Lambda}, \overrightarrow{0}\right)$. Граф $\bar{\Gamma}_{\Lambda}$ дистанциионно-регулярен тогда и только тогда, когда $p_{1 i}^{(t)}\left(\theta_{1}\right)=p_{1 i}^{(t)}\left(\theta_{1}^{\prime}\right)$ для всех $\theta_{1}, \theta_{1}^{\prime} \in V_{n}^{(t)}\left(\bar{\Gamma}_{\Lambda}, \overrightarrow{0}\right)$ и всех $t, i \in \mathbb{N}_{0}$.

Доказательство. Пусть $\alpha, \beta, \delta \in V_{n}$ - такие попарно различные векторы, что $\mu_{\Lambda}(\alpha, \delta)=1, \mu_{\Lambda}(\delta, \beta)=i, \mu_{\Lambda}(\alpha, \beta)=j$ и $\theta_{1}=\alpha \oplus \beta \in W_{n}^{(j)}\left(\bar{\Gamma}_{\Lambda}, \overrightarrow{0}\right)$, $\theta_{2}=\alpha \oplus \delta \in W_{n}^{(1)}\left(\bar{\Gamma}_{\Lambda}, \overrightarrow{0}\right), \theta_{1} \oplus \theta_{2}=\beta \oplus \delta \in W_{n}^{(i)}\left(\bar{\Gamma}_{\Lambda}, \overrightarrow{0}\right)$. Тогда для любых векторов $\alpha^{\prime}, \beta^{\prime}, \delta^{\prime} \in V_{n}$, удовлетворяющих равенствам $\theta_{1}=\alpha^{\prime} \oplus \beta^{\prime}, \theta_{2}=\alpha^{\prime} \oplus \delta^{\prime}$, $\theta_{3}=\beta^{\prime} \oplus \delta^{\prime}=\theta_{1} \oplus \theta_{2}$, выполняются также и равенства $\mu_{\Lambda}\left(\alpha^{\prime}, \delta^{\prime}\right)=1$, $\mu_{\Lambda}\left(\delta^{\prime}, \beta^{\prime}\right)=i, \mu_{\Lambda}\left(\alpha^{\prime}, \beta^{\prime}\right)=j$.

Таким образом, фиксацией векторов $\alpha, \beta$ однозначно задается вектор $\theta_{1}=\alpha \oplus \beta$. Число вершин $\delta \in V_{n}$, для которых $\mu_{\Lambda}(\alpha, \delta)=1, \mu_{\Lambda}(\delta, \beta)=i$, равно числу всех таких векторов $\theta_{2} \in W_{n}^{(i)}\left(\bar{\Gamma}_{\Lambda}, \overrightarrow{0}\right)$, что $\theta_{1} \oplus \theta_{2} \in W_{n}^{(j)}\left(\bar{\Gamma}_{\Lambda}, \overrightarrow{0}\right)$. При этом вектор $\delta$ однозначно задается равенством $\delta=\alpha \oplus \theta_{2}$.

Значит, граф $\bar{\Gamma}_{\Lambda}$ дистанционно-регулярен тогда и только тогда, когда $p_{1 i}^{(j)}\left(\theta_{1}\right)=p_{1 i}^{(j)}\left(\theta_{1}^{\prime}\right)$ для всех $\theta_{1}, \theta_{1}^{\prime} \in W_{n}^{(j)}\left(\bar{\Gamma}_{\Lambda}, \overrightarrow{0}\right)$ и всех $j, i \in \mathbb{N}_{0}$. Лемма доказана.

Пусть $q^{\prime}=(q-1) / 2$ и $\widetilde{p}_{i j}^{(t)}-$ число таких векторов $\delta_{2} \in$ $\in W_{n}^{(i)}\left(\bar{\Gamma}_{1 \cup(q-1)}, \overrightarrow{0}\right)$, что $\delta_{1} \oplus \delta_{2} \in W_{n}^{(j)}\left(\bar{\Gamma}_{1 \cup(q-1)}, \overrightarrow{0}\right)$, где $\delta_{1} \in W_{n}^{(t)}\left(\bar{\Gamma}_{1 \cup(q-1)}, \overrightarrow{0}\right)$. Так как граф $\bar{\Gamma}_{1 \cup(q-1)}$ дистанционно-регулярен, то величина $\widetilde{p}_{i j}^{(t)}$ не зависит от $\delta_{2}$. Величина $\widetilde{p}_{i j}^{(t)}$ называется структурной константой.

Лемма 4. Для $j, t \in\left\{0, \ldots,\left\lceil q^{\prime}\right\rceil\right\}$ структурные константы $\widetilde{p}_{i j}^{(t)}$ графа $\bar{\Gamma}_{1 \cup(q-1)}$ paвнbl:

1) $\widetilde{p}_{1 t-1}^{(t)}=t$ для $t \in\left\{1, \ldots, q^{\prime}\right\}$ и $\widetilde{p}_{1 t-1}^{(t)}=q$ для $t>q^{\prime}$, если $j=t-1$;

2) $\widetilde{p}_{1 t+1}^{(t)}=q-t$, если $j=t+1$;

3) $\widetilde{p}_{1 q^{\prime}}^{\left(q^{\prime}\right)}=q^{\prime}$, если $t=j=q^{\prime}$;

4) $\widetilde{p}_{1 j}^{(t)}=0$ в остальных случаях. 
Доказательство. Положим $\delta_{1}=\varepsilon_{1} \oplus \ldots \oplus \varepsilon_{t}, \lambda=\varepsilon_{1} \oplus \ldots \oplus \varepsilon_{q-1}$.

Пусть $j=t-1$. Если $t \leq\left\lceil q^{\prime}\right\rceil$, то условия $\delta_{1} \oplus \delta_{2} \in W_{n}^{(t-1)}\left(\bar{\Gamma}_{1 \cup(q-1)}, \overrightarrow{0}\right)$ и $\delta_{2} \in\left\{\varepsilon_{1}, \ldots, \varepsilon_{t}\right\}$ эквивалентны. Поэтому $\widetilde{p}_{1 t-1}^{(t)}=t$. Если $t>q^{\prime}$, то условия $\delta_{1} \oplus \delta_{2} \in W_{n}^{(t-1)}\left(\bar{\Gamma}_{1 \cup(q-1)}, \overrightarrow{0}\right)$ и $\delta_{2} \in\left\{\varepsilon_{1}, \ldots, \varepsilon_{q-1}, \lambda\right\}$ эквивалентны. Поэтому $\tilde{p}_{1 j}^{(t)}=q$.

Пусть $j=t+1$. Условия $\delta_{1} \oplus \delta_{2} \in W_{n}^{(t+1)}\left(\bar{\Gamma}_{1 \cup(q-1)}, \overrightarrow{0}\right)$ и $\delta_{2} \in$ $\in\left\{\varepsilon_{t+1}, \ldots, \varepsilon_{q-1}, \lambda\right\}$ эквивалентны. Поэтому $\widetilde{p}_{1 t+1}^{(t)}=q-t$.

Если $t=j=q^{\prime}$, то условия $\delta_{1} \oplus \delta_{2} \in W_{n}^{\left(q^{\prime}\right)}\left(\bar{\Gamma}_{1 \cup(q-1)}, \overrightarrow{0}\right)$ и $\delta_{2} \in$ $\in\left\{\varepsilon_{q^{\prime}+1}, \ldots, \varepsilon_{q-1}, \lambda\right\}$ эквивалентны. Поэтому $\widetilde{p}_{1 q^{\prime}}^{\left(q^{\prime}\right)}=q^{\prime}$.

Аналогичным способом показывается, что во всех остальных случаях $\widetilde{p}_{1 j}^{(t)}=0$. Лемма доказана.

Продолжим доказательство п. 3 теоремы 1. Нетрудно видеть, что

$$
\begin{aligned}
& W_{n}^{(j)}\left(\bar{\Gamma}_{\Theta}, \overrightarrow{0}\right)= \\
& =\left\{\delta_{b_{1}} \oplus \ldots \oplus \delta_{b_{u}} \mid \delta_{b_{i}} \in W_{n, j_{i}, q-j_{i}}^{\left(b_{i}\right)}, j_{1}+\ldots+j_{u}=j, 1 \leq b_{1}<\ldots<b_{u} \leq r\right\},
\end{aligned}
$$

где $j=1, \ldots, r \cdot\left\lceil q^{\prime}\right\rceil$.

Если $r=1$, то $W_{n}^{(j)}\left(\bar{\Gamma}_{\Theta}, \overrightarrow{0}\right)=W_{n}^{(j)}\left(\bar{\Gamma}_{1 \cup(q-1)}, \overrightarrow{0}\right), j=1, \ldots,\lceil(q-1) / 2\rceil$. Значит, $\bar{\Gamma}_{1 \cup(q-1)} \cong \bar{\Gamma}_{\Theta}$, и граф $\bar{\Gamma}_{(\overrightarrow{0}, \gamma)}(g)$ дистанционно-регулярен.

Пусть $r \geq 2$ и $t \in\left\{1, \ldots,\left\lceil q^{\prime}\right\rceil\right\}$. Из леммы 3 следует, что для доказательства дистанционной регулярности графа $\bar{\Gamma}_{\Theta}$ достаточно проверить выполнение условия $p_{1 i}^{(t)}\left(\theta_{1}\right)=p_{1 i}^{(t)}\left(\theta_{1}^{\prime}\right)$ для всех $\theta_{1}, \theta_{1}^{\prime} \in W_{n}^{(t)}\left(\bar{\Gamma}_{\Theta}, \overrightarrow{0}\right), t, i \in \mathbb{N}_{0}$.

Очевидно, что

$$
W_{n}^{(t)}\left(\bar{\Gamma}_{\Theta}, \overrightarrow{0}\right) \oplus \Theta \subseteq W_{n}^{(t-1)}\left(\bar{\Gamma}_{\Theta}, \overrightarrow{0}\right) \cup W_{n}^{(t)}\left(\bar{\Gamma}_{\Theta}, \overrightarrow{0}\right) \cup W_{n}^{(t+1)}\left(\bar{\Gamma}_{\Theta}, \overrightarrow{0}\right),
$$

где $W_{n}^{(1)}\left(\bar{\Gamma}_{\Theta}, \overrightarrow{0}\right)=\Theta$. Значит, $p_{1 t-c}^{(t)}(\delta)=p_{1 t+c}^{(t)}(\delta)=0$ для любых $\delta \in V_{n}$ и $c \geq 2$.

Из задания множества $W_{n, j, q-j}^{\left(b_{1}\right)}$ следует, что $W_{n, j, q-j}^{\left(b_{1}\right)} \cap W_{n, i, q-i}^{\left(b_{2}\right)}=\varnothing$, если $b_{1} \neq b_{2}$ или $i \neq j$. Таким образом, для произвольного вектора $\delta \in W_{n}^{(t)}\left(\bar{\Gamma}_{\Theta}, \overrightarrow{0}\right)$, представимого в виде $\delta=\delta_{1} \oplus \ldots \oplus \delta_{r}, \delta_{i} \in W_{n, t_{i}, q-t_{i}}^{(i)}$, и произвольных чисел $j, t \in \mathbb{N}$ выполняется равенство

$$
p_{1 j}^{(t)}(\delta)=\sum_{\substack{j_{1}+\ldots+j_{r}=j, j_{c} \in\{0, \ldots, j\}, c=1, \ldots, r}}\left(\widetilde{p}_{1_{j_{1}}}^{\left(t_{1}\right)}+\ldots+\widetilde{p}_{1_{j_{r}}}^{\left(t_{r}\right)}\right), \quad t_{1}+\ldots+t_{r}=t .
$$


Рассмотрим такое максимальное подмножество $\left\{b_{1}, \ldots, b_{u}\right\} \subseteq\{1, \ldots, r\}$, что $t_{b_{c}}>0$ для $c=1, \ldots, u$. В этом случае, $\delta=\delta_{b_{1}} \oplus \ldots \oplus \delta_{b_{u}}, t_{b_{1}}+\ldots+t_{b_{u}}=t$. получаем

Пусть $t_{b_{i}}<\left\lceil q^{\prime}\right\rceil, i=1, \ldots, u$. Тогда из леммы 4 и равенства (2)

$$
\begin{gathered}
p_{1 t+1}^{(t)}(\delta)=\widetilde{p}_{1\left(t_{b_{1}}+1\right)}^{\left(t_{b_{1}}\right)}+\ldots+\widetilde{p}_{1\left(t_{b_{u}}+1\right)}^{\left(t_{b_{b}}\right)}+\widetilde{p}_{11}^{(0)}(r-u)=\sum_{i=1}^{u}\left(-t_{b_{i}}\right)+q r=q r-t \\
p_{1 t-1}^{(t)}(\delta)=\widetilde{p}_{1\left(t_{b_{1}}-1\right)}^{\left(t_{b_{1}}\right)}+\ldots+\widetilde{p}_{1\left(t_{b_{u}}-1\right)}^{\left(t_{b_{u}}\right)}=\sum_{i=1}^{u} t_{b_{i}}=t, \quad p_{1 t}^{(t)}(\delta)=0
\end{gathered}
$$

Пусть $q-1$ четно. Если $t_{c_{1}}=\ldots=t_{c_{v}}=q^{\prime}, t_{i}<q^{\prime}$ для всех $i \in$ $\in\left\{b_{1}, \ldots, b_{u}\right\} \backslash\left\{c_{1}, \ldots, c_{v}\right\}$, то из леммы 4 и равенства (2) имеем

$$
p_{1 t}^{(t)}(\delta)=\widetilde{p}_{1 t_{b_{1}}}^{\left(t_{b_{1}}\right)}+\ldots+\widetilde{p}_{1 t_{b_{u}}}^{\left(t_{b_{u}}\right)}=v q^{\prime}, \quad p_{1(t-1)}^{(t)}(\delta)=\widetilde{p}_{1\left(t_{b_{1}}-1\right)}^{\left(t_{b_{1}}\right)}+\ldots+\widetilde{p}_{1\left(t_{b_{u}}-1\right)}^{\left(t_{b_{u}}\right)}=t .
$$

Пусть $r \geq 2$ и $q>3$. Пусть $t=q^{\prime}+1$ и $\delta, \delta^{\prime}-$ такие векторы из $W_{n}^{(t)}\left(\bar{\Gamma}_{\Theta}, \overrightarrow{0}\right)$, что $\delta=\delta_{1} \oplus \delta_{2}$ и $\delta^{\prime}=\delta_{1}^{\prime} \oplus \delta_{2}^{\prime}, \delta_{1} \in V_{n, q^{\prime}, q^{\prime}+1}^{(2)}, \delta_{2} \in V_{n, 1, q-1}^{(2)}$ и $\delta_{1}^{\prime} \in V_{n, j_{1}, q-j_{1}}^{(2)}, \delta_{2}^{\prime} \in V_{n, j_{2}, q-j_{2}}^{(2)}, j_{1}+j_{2}=t, j_{1}, j_{2}<q^{\prime}$. Из равенств (4) и (5) получаем: $p_{1 t}^{(t)}(\delta)=q^{\prime}, p_{1 t}^{(t)}\left(\delta^{\prime}\right)=0$. Из леммы 3 и изоморфизма $\bar{\Gamma}_{(\overrightarrow{0}, \gamma)}(g) \cong \bar{\Gamma}_{\Theta}$ следует, что граф $\bar{\Gamma}_{\Theta}$ не дистанционно-регулярен.

Пусть $r \geq 2$ и $q=3$. Тогда из леммы 4 следует, что $\widetilde{p}_{11}^{(1)}=2, \widetilde{p}_{11}^{(0)}=$ $=3, \widetilde{p}_{10}^{(1)}=1, \widetilde{p}_{10}^{(0)}=0$ и $\widetilde{p}_{1 j}^{(t)}=0$, если $\{t, j\} \nsubseteq\{0,1\}$. Таким образом, для произвольного вектора вида $\delta=\delta_{b_{1}} \oplus \ldots \oplus \delta_{b_{u}} \in W_{n}^{(t)}\left(\bar{\Gamma}_{\Theta}, \overrightarrow{0}\right)$, где $\delta_{b_{i}} \in V_{n, 1,1}^{\left(b_{i}\right)}$, и $t_{b_{1}}=\ldots=t_{b_{u}}=1$ равенство (2) принимает вид

$$
\begin{gathered}
p_{1 t}^{(t)}(\delta)=\widetilde{p}_{1 t_{b_{1}}}^{\left(t_{b_{1}}\right)}+\ldots+\widetilde{p}_{1 t_{b_{u}}}^{\left(t_{b_{u}}\right)}=\widetilde{p}_{11}^{(1)}+\ldots+\widetilde{p}_{11}^{(1)}=2 t, \\
p_{1(t-1)}^{(t)}(\delta)=\widetilde{p}_{1\left(t_{b_{1}}-1\right)}^{\left(t_{b_{1}}\right)}+\ldots+\widetilde{p}_{1\left(t_{b_{u}}-1\right)}^{\left(t_{b_{u}}\right)}=\widetilde{p}_{10}^{(1)}+\ldots+\widetilde{p}_{10}^{(1)}=t, \\
p_{1(t+1)}^{(t)}(\delta)=(r-t) \cdot \tilde{p}_{11}^{(0)}=3 \cdot(r-t) .
\end{gathered}
$$

Из леммы 3 следует, что граф $\bar{\Gamma}_{\Theta}$ дистанционно-регулярен. Так как $\bar{\Gamma}_{(\overrightarrow{0}, \gamma)}(g) \cong$ $\cong \bar{\Gamma}_{\Theta}$, то $\bar{\Gamma}_{(\overrightarrow{0}, \gamma)}(g)$ дистанционно-регулярен.

Пусть теперь $q-1$ нечетно. Если $t_{c_{1}}=\ldots=t_{c_{v}}=\left\lceil q^{\prime}\right\rceil=q / 2$ и $t_{b_{i}}<q / 2$ для всех $i \in\left\{b_{1}, \ldots, b_{u}\right\} \backslash\left\{c_{1}, \ldots, c_{v}\right\}$, то $t=v q / 2+\sum_{i \in\left\{b_{1}, \ldots, b_{u}\right\} \backslash\left\{c_{1}, \ldots, c_{v}\right\}} t_{i}$. Из 
равенств (2) и леммы 4 имеем $p_{1 t}^{(t)}(\delta)=0$,

$$
p_{1(t-1)}^{(t)}(\delta)=\widetilde{p}_{1\left(t_{b_{1}}-1\right)}^{\left(t_{b_{1}}\right)}+\ldots+\widetilde{p}_{1\left(t_{b_{u}}-1\right)}^{\left(t_{b_{u}}\right)}=v q+\sum_{i \in\left\{b_{1}, \ldots, b_{u}\right\} \backslash\left\{c_{1}, \ldots, c_{v}\right\}} t_{i}=t+v q / 2 .
$$

Пусть $q>2$. Пусть $t=q / 2+1$ и векторы $\delta, \delta^{\prime} \in V_{n}^{(t)}\left(\bar{\Gamma}_{\Theta}, \overrightarrow{0}\right)$ удовлетворяют условиям $\delta=\delta_{1} \oplus \delta_{2}, \delta^{\prime}=\delta_{1}^{\prime} \oplus \delta_{2}^{\prime}, \delta_{1} \in V_{n, q / 2, q / 2}^{(2)}, \delta_{2} \in V_{n, 1, q-1}^{(2)}$ и $\delta_{1}^{\prime} \in V_{n, j_{1}, q-j_{1}}^{(2)}, \delta_{2}^{\prime} \in V_{n, j_{2}, q-j_{2}}^{(2)}, j_{1}+j_{2}=t, j_{1}, j_{2}<q / 2$. Тогда из равенств (4) и (6) получаем

$$
p_{1(t-1)}^{(t)}(\delta)=t+q / 2=q+1, \quad p_{1(t-1)}^{(t)}\left(\delta^{\prime}\right)=q / 2+1
$$

Из леммы 3 и изоморфизма $\bar{\Gamma}_{(\overrightarrow{0}, \gamma)}(g) \cong \bar{\Gamma}_{\Theta}$ следует, что граф $\bar{\Gamma}_{\Theta}$ не дистанционно-регулярен.

Если $q=2$, то $m_{\gamma, g}(x)=x^{r} \oplus 1$. Значит, $m=r$. Однако $m=r q$, что невозможно. Теорема доказана.

Отметим, что у ряда $(n \times n)$-матриц, используемых в блочных шифрсистемах, минимальный многочлен имеет вид $(x \oplus 1)^{r}$. Кроме того, если $r=1$ в п. 3 теоремы 1 , то из доказательства теоремы 1 следует, что граф $\bar{\Gamma}_{(\overrightarrow{0}, \gamma)}(g)$ изоморфен графу $\bar{\Gamma}_{1 \cup n}$.

Рассмотрим случай, когда $r \geq 2$ и $q=3$. Тогда матрица преобразования $g^{r}$ подобна клеточно-диагональной матрице $\mathrm{c} r$ диагональными порядка три клетками $a_{1}=\ldots=a_{r}=\left(\begin{array}{ll}0 & 1 \\ 1 & 1\end{array}\right)$. При $r=2$ и $q=3$ получаем подобие матриц:

$$
g \approx\left(\begin{array}{llll}
0 & 1 & 0 & 0 \\
0 & 0 & 1 & 0 \\
0 & 0 & 0 & 1 \\
1 & 0 & 1 & 0
\end{array}\right)
$$

В общем случае $(r \geq 2, q=3)$ матрица преобразования $g$ подобна $(2 r \times$ $\times 2 r)$-матрице $a=\left(a_{i j}\right)$, где

$$
a_{i j}=\left\{\begin{array}{l}
1, \text { если } j=i+1, i \in\{1, \ldots, n-1\} \\
1, \text { если } i=n, j \in\{1, r+1\} \\
0 \text { в остальных случаях. }
\end{array}\right.
$$

При этом

$$
m_{a}(x)=\operatorname{HOK}\left(m_{\varepsilon_{1}, a}(x), \ldots, m_{\varepsilon_{n}, a}(x)\right)=x^{n} \oplus x^{n / 2} \oplus 1=u_{a}(x) .
$$




\section{5. Метрики графов орбиталов группы $C(g)$}

В зависимости от структуры преобразования $g \in G L_{n}$ и вектора $\gamma \in V_{n}^{\times}$ возникают как надметрики, так и подметрики метрики Хемминга $\chi_{n}$. Выведем условие, при котором среди метрик графа орбитала группы $C(g)$ присутствует метрика Хемминга.

Утверждение 6. Пусть $g \in G L_{n}$ u $M(g)$ - множество всех нетривиальных метрик графов орбиталов группы $C(g)$. Метрика $\chi_{n} \in M(g)$ тогда и только тогда, когда $g$ - подстановочная матрица, которой соответствует полночикловая подстановка на множестве $\{0, \ldots, n-1\}$.

Доказательство. Если $\chi_{n} \in M(g)$, то граф связен и по следствию 1 существует такой вектор $\gamma \in V_{n}$, что

$$
V_{n}^{(1)}(g, \gamma)=\gamma^{\langle g\rangle}=\Delta_{1}^{(n)}
$$

Следовательно, $\varepsilon_{i}^{g} \in \Delta_{1}^{(n)}$ для любого $i \in\{0, \ldots, n-1\}$, т. е. $g-$ подстановочная матрица. Так как $\left|\gamma^{\langle g\rangle}\right|=n$, то ord $(g)=n$ и $g$ соответствует полноцикловая подстановка. Утверждение доказано.

Утверждение 7. Пусть $\gamma \in V_{n}^{\times}, g \in G L_{n}$. Метрика $\mu_{\gamma, g} \cong \chi_{n}$ тогда и только тогда, когда $\gamma^{\langle g\rangle}-$ базис пространства $V_{n}$.

Доказательство следует из утверждения 1 и следствия 1.

Укажем связи между метриками графов орбиталов группы $C(g)$ и надгруппами группы Джевонса из работы [5].

Опишем метрики графов орбиталов группы $C(g)$, являющиеся надметриками или подметриками метрики Хемминга. Отметим, что частичная классификация подметрик и надметрик метрики Хемминга приведена в $[6,10]$.

Лемма 5. 1. Не существует преобразования $g \in G L_{n}$ и вектора $\gamma \in V_{n}^{\times}$, для которых граф $\bar{\Gamma}_{(\overrightarrow{0}, \gamma)}(g)$ связен $и \mu_{\gamma, g}-$ надметрика метрики Хемминга $\chi_{n}$, не совпадающиа с $\chi_{n}$.

2. Пусть $\gamma \in \Delta_{i}^{(n)}$ и граф $\bar{\Gamma}_{(\overrightarrow{0}, \gamma)}(g)$ связен. Метрика $\mu_{\gamma, g}$ тогда и только тогда является подметрикой метрики $\chi_{n}$, когда $\bigcup_{j=1}^{c} \Delta_{j}^{(n)}=\gamma^{\langle g\rangle}$ для некоторого $\mathrm{c} \in\{i, \ldots, n\}$.

Доказательство. 1. Предположим, что $\mu_{\gamma, g}-$ надметрика метрики Хемминга $\chi_{n}$ для некоторого $g \in G L_{n}$. Тогда $V_{n}^{(i)}(g, \gamma)=\Delta_{i}^{(n)}$ и $V_{n}^{(t)}(g, \gamma) \subset$ $\subset \Delta_{t}^{(n)}$ для некоторых $t \in\{1, \ldots, n-1\}$ и $i=1,2, \ldots, t-1$. Если $t=1$ 
и $V_{n}^{(1)}(g, \gamma) \subset \Delta_{1}^{(n)}$, то из следствия 1 получаем несвязность графа $\bar{\Gamma}_{(\overrightarrow{0}, \gamma)}(g)$. Если $t \geq 2$, то из равенства $V_{n}^{(1)}(g, \gamma)=\Delta_{1}^{(n)}$ и утверждения 1 следует, что $V_{n}^{(i)}(g, \gamma)=\Delta_{i}^{(n)}, i=1,2, \ldots, t$.

П. 2 непосредственно следует из определения подметрики и утверждения 1. Лемма доказана.

Приведем пример несвязного графа $\bar{\Gamma}_{(\overrightarrow{0}, \gamma)}(g)$, у которого существуют компоненты связности, изоморфные графу Хемминга.

ПРИМЕР 1. Пусть $g$ - подстановочная матрица, не являющаяся полноцикловой подстановкой на множестве $\{0, \ldots, n-1\}, \gamma \in \Delta_{1}^{(n)}, r=$ $=\operatorname{deg} m_{\gamma, g}(x)=\left|\gamma^{\langle g\rangle}\right|$. Тогда граф $\bar{\Gamma}_{(\overrightarrow{0}, \gamma)}(g)$ состоит из $2^{n-r}$ изоморфных компонент связности, а метрика каждой компоненты связности графа $\bar{\Gamma}_{(\overrightarrow{0}, \gamma)}(g)$ изоморфна метрике Хемминга $\chi_{r}$ на $V_{r}$.

Если $G \leq S\left(V_{n}\right),\{\overrightarrow{0}\} \times \Delta_{n}^{(1)} \subseteq(\alpha, \beta)^{G}$, то орбитал $(\alpha, \beta)^{G}$ называется базовым (см. [6]). Пусть $R-$ надгруппа группы подстановочных матриц $\widetilde{S}_{n}, \rho_{R}-$ базовая метрика, т. е. метрика базового орбитала группы $R$, и $g-$ произвольное преобразование из $G L_{n}$,

$$
\begin{array}{cc}
h_{1, n}= & \left(\begin{array}{cccc}
1 & 0 & \cdots & 0 \\
1 & 1 & & \mathbf{0} \\
\vdots & & \ddots & \\
1 & \mathbf{0} & & 1
\end{array}\right), \quad h_{2, n}=\left(\begin{array}{cccc}
1 & 1 & \cdots & 1 \\
0 & 1 & & \mathbf{0} \\
\vdots & & \ddots & \\
0 & \mathbf{0} & & 1
\end{array}\right), \\
S_{n}^{(1)}=\left\langle\widetilde{S}_{n}, h_{1, n}\right\rangle, & R_{n}^{(1)}=\left\langle\widetilde{S}_{n}, h_{2, n}\right\rangle .
\end{array}
$$

Группы $R_{n}^{(1)}, S_{n}^{(1)}$, возникшие в [5], изоморфны группе $\widetilde{S}_{n+1}$, и являются наименьшими надгруппами группы $\widetilde{S}_{n}$. Группы $A R_{n}^{(1)}, A S_{n}^{(1)}$, порожденные соответственно группами $R_{n}^{(1)}, S_{n}^{(1)}$ и группой сдвигов, являются наименьшими надгруппами группы Джевонса. Опишем свойства их базовых метрик.

Для произвольных $i \in\{0, \ldots, n-1\}, g \in G L_{n}$ положим

$$
n_{i}(g)=\left|\left\{\bar{\Gamma}_{(\overrightarrow{0}, \gamma)}(g) \mid \gamma \in \Delta_{i}^{(n)}\right\}\right| .
$$

Утверждение 8. Если $n \geq 3$, то:

1. Для каждого преобразования $g \in S_{n}^{(1)} \backslash \widetilde{S}_{n}$ существует такая пара $\left(j, r_{g}\right) \in\{0, \ldots, n-1\} \times S_{n}$, что

$$
\begin{aligned}
g & :\left(\alpha_{n-1}, \ldots, \alpha_{0}\right) \rightarrow \\
& \rightarrow\left(\alpha_{(n-1)^{r_{g}}}, \ldots, \alpha_{(j+1)^{r_{g}}}, \alpha_{0} \oplus \ldots \oplus \alpha_{n-1}, \alpha_{(j-1)^{r_{g}}}, \ldots, \alpha_{0^{r_{g}}}\right) .
\end{aligned}
$$


2. Если g, g' - различные преобразования из $S_{n}^{(1)} \backslash \widetilde{S}_{n}$, то:

2.1. Графы орбиталов групп $C(g)$ и $C\left(g^{\prime}\right)$ изоморфны тогда и только тогда, когда подстановки $r_{g}, r_{g^{\prime}}$ имеют одинаковую иикловую структуру.

2.2. Если подстановка $r_{g}$ имеет неподвижные точки, то все графы орбиталов группь $C(g)$ не связны.

2.3. Если подстановка $r_{g}$ является произведением $t \geq 2$ независимых цииклов, то для любого вектора $\gamma \in \bigcup_{i=1}^{t-1} \Delta_{i}^{(n)}$ граф $\bar{\Gamma}_{(\overrightarrow{0}, \gamma)}(g)$ не связен.

3. Не существует пары $(\gamma, g) \in\left(V_{n}^{\times}, S_{n}^{(1)} \backslash \widetilde{S}_{n}\right)$, удовлетворяющей равенсmвy $\rho_{S_{n}^{(1)}}=\mu_{\gamma, g}$.

4. Если $g \in R_{n}^{(1)} \backslash \widetilde{S}_{n}$ и подстановка $r_{g} \in S_{n}$ полноцикловая, то $n_{1}(g)=n / 2$ при четном $п$ и все графы из множества $\left\{\bar{\Gamma}_{(\overrightarrow{0}, \gamma)}(g) \mid \gamma \in \Delta_{1}^{(n)}\right\}$ связны; $n_{1}(g)=(n+1) / 2$ при нечетном $n$ и число связных графов из множества $\left\{\bar{\Gamma}_{(\overrightarrow{0}, \gamma)}(g) \mid \gamma \in \Delta_{1}^{(n)}\right\}$ равно $(n-1) / 2$.

Доказательство. П. 1 непосредственно следует из задания группы $S_{n}^{(1)}$ системой образующих $\left\langle\widetilde{S}_{n}, h_{1, n}\right\rangle$ и равенства $h_{1, n}^{2}=e_{n}$.

2. Пусть подстановка $\widetilde{S}$ и соответствующая подстановочная матрица $s$ связаны соотношением

$$
\left(\alpha_{n-1}, \ldots, \alpha_{0}\right)^{s}=\left(\alpha_{(n-1)^{\widetilde{s}}}, \ldots, \alpha_{0^{\widetilde{s}}}\right)
$$

Пусть $g=s_{1} h_{1, n} s_{2}$ для некоторых подстановочных матриц $s_{1}, s_{2}$. Тогда

$$
\left(\alpha_{n-1}, \ldots, \alpha_{0}\right)^{s_{1} h_{1, n}}=\left(\alpha_{n-1} \oplus \ldots \oplus \alpha_{0}, \alpha_{(n-2)^{\widetilde{s}_{1}}}, \ldots, \alpha_{0^{\widetilde{s}_{1}}}\right)
$$

и

$$
\begin{aligned}
& \left(\alpha_{n-1}, \ldots, \alpha_{0}\right)^{g}= \\
& =\left(\alpha_{i}, \alpha_{(n-2)^{\widetilde{s}_{1}} \widetilde{s}_{2}}, \ldots, \alpha_{(j+1)^{\widetilde{s}_{1} \widetilde{s}_{2}}}, \alpha_{n-1} \oplus \ldots \oplus \alpha_{0}, \alpha_{(j-1)^{\widetilde{s}_{1} \widetilde{s}_{2}}}, \ldots, \alpha_{0^{\widetilde{s}_{1} \widetilde{s}_{2}}}\right) \text {, }
\end{aligned}
$$

где $\tilde{i}^{\widetilde{s}_{1}}=j$ и $\tilde{j}^{\tilde{s}_{2}}=n-1$. 
Значит, преобразование $g=s_{1} h_{1, n} s_{2}$ однозначно задается такой парой $\left(j, r_{g}\right) \in\{0, \ldots, n-1\} \times S_{n}$, что

$$
\left(\alpha_{n-1}, \ldots, \alpha_{0}\right)^{g}=\left(\alpha_{(n-1)^{r_{g}}}, \ldots, \alpha_{(j+1)^{r_{g}}}, \alpha_{0} \oplus \ldots \oplus \alpha_{n-1}, \alpha_{(j-1)^{r^{g}}}, \ldots, \alpha_{0^{r^{g}}}\right)
$$

Кроме того, каждая пара $\left(j, r_{g}\right)$ однозначно задает некоторый элемент группы $S_{n}^{(1)}$, что дает взаимно однозначное соответствие $\varphi:\{0, \ldots, n-1\} \times S_{n} \rightarrow$ $\rightarrow S_{n}^{(1)}$ и

$$
S_{n, 1}^{(1)}=\left\{\varphi\left(j, r_{g}\right) \mid\left(j, r_{g}\right) \in\{0, \ldots, n-1\} \times S_{n}\right\} .
$$

Ясно, что $S_{n, 1}^{(1)} \subset S_{n}^{(1)}, S_{n, 1}^{(1)} \cap \widetilde{S}_{n}=\varnothing$. Учитывая равенства $\left|S_{n, 1}^{(1)}\right|=n ! n,\left|\widetilde{S}_{n}\right|=$ $=n !,\left|S_{n}^{(1)}\right|=(n+1) !,\left|S_{n, 1}^{(1)}\right|+\left|\widetilde{S}_{n}\right|=\left|S_{n}^{(1)}\right|$, из количественных соображений получаем, что $S_{n, 1}^{(1)} \cup \widetilde{S}_{n}=S_{n}^{(1)}$.

Значит, каждое преобразование $g \in S_{n}^{(1)} \backslash \widetilde{S}_{n}$ однозначно задается парой $\left(j, r_{g}\right) \in\{0, \ldots, n-1\} \times S_{n}$. Теперь п. 2 следует из п. 1 .

Пп. 3 и 4. Если $\rho_{S_{n}^{(1)}}=\mu_{\gamma, g}$ для некоторых $\gamma \in V_{n}^{\times}$и $g \in S_{n}^{(1)} \backslash \widetilde{S}_{n}$, то

$$
V_{n}^{(1)}(g, \gamma)=V_{n}^{(1)}\left(\rho_{S_{n}^{(1)}}\right)=\gamma^{\langle g\rangle}=\Delta_{1}^{(n)} \cup \Delta_{2}^{(n)}
$$

При этом $\left|V_{n}^{(1)}\left(\rho_{S_{n}^{(1)}}\right)\right|=n+\left(\begin{array}{c}n \\ 2\end{array}\right)$.

Пусть $j=0, r_{g}=(n-1, \ldots, 1,0),\left(\alpha_{n-1}, \ldots, \alpha_{0}\right)^{g}=\left(\alpha_{n-2}, \ldots, \alpha_{0}, \alpha_{0} \oplus\right.$ $\left.\ldots \oplus \alpha_{n-1}\right), t=0, \ldots,\lceil n / 2\rceil$. Тогда

$\varepsilon_{t}^{\langle g\rangle}=V_{n}^{(1)}\left(g, \varepsilon_{t}\right)=\left\{\varepsilon_{t}\right\} \cup\left\{\varepsilon_{n-t-1}\right\} \cup\left(\bigcup_{i=0}^{n-t-1}\left\{\varepsilon_{i} \oplus \varepsilon_{i+t}\right\}\right) \cup\left(\bigcup_{i=0}^{t-1}\left\{\varepsilon_{n-t+i} \oplus \varepsilon_{i}\right\}\right)$.

Если $n$ четно, то $\left|V_{n}^{(1)}\left(g, \varepsilon_{t}\right)\right|=n+1$ и $\left\langle\varepsilon_{t}^{\langle g\rangle}\right\rangle=V_{n}$, т. е. все графы множества $\left\{\bar{\Gamma}_{(\overrightarrow{0}, \gamma)}(g) \mid \gamma \in \Delta_{1}^{(n)}\right\}$ связны.

Если $n$ нечетно и $t=(n+1) / 2$, то

$$
\varepsilon_{t}^{\langle g\rangle}=V_{n}^{(1)}\left(g, \varepsilon_{t}\right)=\left\{\varepsilon_{t}\right\} \cup\left(\bigcup_{i=0}^{n-t-1}\left\{\varepsilon_{i} \oplus \varepsilon_{i+t}\right\}\right)
$$

т. е. $\left|V_{n}^{(1)}\left(g, \varepsilon_{t}\right)\right|=(n+1) / 2$. Если $t \in\{1, \ldots,(n-1) / 2\}$, то $\left|V_{n}^{(1)}\left(g, \varepsilon_{t}\right)\right|=$ $=n+1$ и граф $\bar{\Gamma}_{\left(\overrightarrow{0}, \varepsilon_{t}\right)}(g)$ связен. 
Следовательно, для любого вектора $\gamma \in \Delta_{1}^{(n)}$ имеем $\left|V_{n}^{(1)}(g, \gamma)\right|<$ $<\left|V_{n}^{(1)}\left(\rho_{S_{n}^{(1)}}\right)\right|$. Из пп. 1 и 2 следует, что если $r_{g}-$ произвольная полноцикловая подстановка и преобразование $g$ задается равенством (7), то $\left|V_{n}^{(1)}(g, \gamma)\right|<\left|V_{n}^{(1)}\left(\rho_{S_{n}^{(1)}}\right)\right|$ для любого вектора $\gamma \in \Delta_{1}^{(n)}$.

Пусть теперь подстановка $r_{g}$ не полноцикловая. Из п. 2 утверждения 8 следует, что граф $\bar{\Gamma}_{(\overrightarrow{0}, \gamma)}(g)$ не связен и $\left|V_{n}^{(1)}(g, \gamma)\right| \leq n$, если $\gamma \in \Delta_{1}^{(n)}$. Следовательно, $\rho_{S_{n}^{(1)}} \neq \mu_{\gamma, g}$ для всех $\gamma \in V_{n}^{\times}$и $g \in S_{n}^{(1)} \backslash \widetilde{S}_{n}$. Утверждение доказано.

Пусть метрика $\rho$ на множестве $V_{n}$ инвариантна относительно группы сдвигов, т. е. $\rho(\alpha, \theta)=\rho(\alpha \oplus \beta, \theta \oplus \beta)$ для всех $\alpha, \theta, \beta \in V_{n}$. Положим $\|\alpha\|_{\rho}=$ $=\rho(\alpha, 0)$ и $\|\alpha\|=\|\alpha\|_{\chi_{n}}$ при $\alpha \in V_{n}$.

Коэффициент рассеивания (branch number) (см. [14, 15]) линейного преобразования $a \in G L_{n}(2)$ и метрики Хемминга $\chi_{n}$ естественным образом обобщается на произвольную метрику $\rho$ на пространстве $V_{n}$ следующим образом:

$$
b_{\rho}(a)=\min \left\{\|\alpha\|_{\rho}+\left\|\alpha^{\alpha}\right\|_{\rho} \mid \alpha \in V_{n}^{\times}\right\} .
$$

Величина $b_{\rho}(a)$ также отражает степень рассеивания преобразования $a$ и используется, например, в разностном методе и его обобщениях. В разностном методе часто используется метрика Хемминга, в методе усеченных разностей вектор разбивается на блоки и рассматривается подметрика метрики Хемминга. При обосновании стойкости XSL-шифрсистем значение $b_{\chi_{n}}(a)$ стараются сделать максимальным.

Нетрудно убедиться, что любое преобразование $g \in S_{n}^{(1)}$ имеет наименьшее возможное значение величины $b_{\chi_{n}}(g)$, равное двум. Действительно, пусть

$$
\left(\alpha_{n-1}, \ldots, \alpha_{0}\right)^{g}=\left(\alpha_{(n-1)^{r g}}, \ldots, \alpha_{(j+1)^{r_{g}}}, \alpha_{0} \oplus \ldots \oplus \alpha_{n-1}, \alpha_{(j-1)^{r_{g}}}, \ldots, \alpha_{0^{r_{g}}}\right)
$$

для некоторых $\left(j, r_{g}\right) \in\{0, \ldots, n-1\} \times S_{n}$. Тогда $\varepsilon_{j}^{g}=\varepsilon_{j}$. Значит, $b_{\chi_{n}}(g)=2$.

Утверждение 9. Если $n \geq 3$, то:

1. Для каждого преобразования $g \in R_{n}^{(1)} \backslash \widetilde{S}_{n}$ существует такая пара $\left(j, r_{g}\right) \in\{0, \ldots, n-1\} \times S_{n}$, что

$$
\begin{aligned}
& \left(\alpha_{n-1}, \ldots, \alpha_{0}\right)^{g}= \\
& =\left(\alpha_{(n-1)^{r g}} \oplus \alpha_{j^{r} g}, \ldots, \alpha_{(j+1)^{r g}} \oplus \alpha_{j^{r} g}, \alpha_{j^{r g}}, \alpha_{(j-1)^{r_{g}}} \oplus \alpha_{j^{r_{g}}}, \ldots, \alpha_{0^{r_{g}}} \oplus \alpha_{j^{r_{g}}}\right) .
\end{aligned}
$$


2. Пусть $g, g^{\prime} \in R_{n}^{(1)} \backslash \widetilde{S}_{n}, g^{\prime} \neq$ g. Графы орбиталов групn $C(g)$ и $C\left(g^{\prime}\right)$ изоморфны тогда и только тогда, когда подстановки $r_{g}, r_{g^{\prime}}$ имеют одинаковую иикловую структуру.

3. Если $\gamma \in V_{n}^{\times}$и преобразованию $g \in R_{n}^{(1)} \backslash \widetilde{S}_{n}$ соответствует полноцикловая подстановка $r_{g} \in S_{n}$, то $\rho_{R_{n}^{(1)}}=\mu_{\gamma, g}$. Кроме того, $n_{1}(g)=1$ и граф $\bar{\Gamma}_{\left(\overrightarrow{0}, \varepsilon_{0}\right)}(g)$ связен.

Доказательство. Доказательство п. 1 непосредственно следует из задания группы $R_{n}^{(1)}$ системой образующих $\left\langle\widetilde{S_{n}}, h_{2, n}\right\rangle$ и полностью аналогично доказательству п. 1 утверждения 8. Значит, каждое преобразование $g \in R_{n}^{(1)} \backslash \widetilde{S}_{n}$ однозначно задается парой $\left(j, r_{g}\right) \in\{0, \ldots, n-1\} \times S_{n}$.

П. 2 следует из п. 1.

3. Отметим, что если $\rho_{R_{n}^{(1)}}=\mu_{\gamma, g}$ для некоторых $\gamma \in V_{n}^{\times}$и $g \in R_{n}^{(1)} \backslash \widetilde{S}_{n}$, To

$$
V_{n}^{(1)}(g, \gamma)=V_{n}^{(1)}\left(\rho_{R_{n}^{(1)}}\right)=\gamma^{\langle g\rangle}=\Delta_{1}^{(n)} \cup \Delta_{n}^{(n)},
$$

причем $\left|V_{n}^{(1)}\left(\rho_{R_{n}^{(1)}}\right)\right|=n+1$.

Пусть $r_{g}=(n-1, n-2, \ldots, 1,0), j=0$ и

$$
\left(\alpha_{n-1}, \ldots, \alpha_{0}\right)^{g}=\left(\alpha_{n-2} \oplus \alpha_{n-1}, \alpha_{n-3} \oplus \alpha_{n-1}, \ldots, \alpha_{0} \oplus \alpha_{n-1}, \alpha_{n-1}\right) .
$$

Тогда $\varepsilon_{0}^{\langle g\rangle}=\Delta_{1}^{(n)} \cup \Delta_{n}^{(n)}$. Значит, $V_{n}^{(1)}(g, \gamma)=V_{n}^{(1)}\left(\rho_{R_{n}^{(1)}}\right)$ и $\rho_{R_{n}^{(1)}}=\mu_{\gamma, g}$. Так как $\Delta_{1}^{(n)} \subset \gamma^{\langle g\rangle}$, то $n_{1}(g)=1$.

Из пп. 1 и 2 следует, что если $g \in R_{n}^{(1)} \backslash \widetilde{S}_{n}, r_{g} \in S_{n}$ и $\gamma \in \Delta_{1}^{(n)}$, то $V_{n}^{(1)}(g, \gamma)=V_{n}^{(1)}\left(\rho_{R_{n}^{(1)}}\right)$, т. е. $\rho_{R_{n}^{(1)}}=\mu_{\gamma, g}$. Аналогично получаем, что $n_{1}(g)=1$. Утверждение доказано.

Если $g \in R_{n}^{(1)}$, то $b_{\chi_{n}}(g) \leq 2$. Действительно, пусть

$$
\begin{aligned}
& \left(\alpha_{n-1}, \ldots, \alpha_{0}\right)^{g}= \\
& =\left(\alpha_{(n-1)^{r g}} \oplus \alpha_{j^{r g}}, \ldots, \alpha_{(j+1)^{r_{g}}} \oplus \alpha_{j^{r g}}, \alpha_{j^{r g}}, \alpha_{(j-1)^{r g}} \oplus \alpha_{j^{r g}}, \ldots, \alpha_{0^{r_{g}}} \oplus \alpha_{j^{r g}}\right)
\end{aligned}
$$

для некоторой пары $\left(j, r_{g}\right) \in\{0, \ldots, n-1\} \times S_{n}$. Тогда $\varepsilon_{i}^{g}=\varepsilon_{i}^{r_{g}}, i=0, \ldots, n-$ $-1, i \neq j$. Значит, $b_{\chi_{n}}(g)=2$.

Укажем связь коэффициента рассеивания $b_{\chi_{n}}$ с базовыми подметриками метрики Хемминга. Пусть $R-$ надгруппа группы Джевонса [6], $g \in R, \Gamma_{R}-$ орбитал группы $R,\left(0, \varepsilon_{0}\right) \in \Gamma_{R}, \mu_{R}-$ метрика графа $\bar{\Gamma}_{R}$,

$$
U_{R}=\left\{i \in\{1, \ldots, n\} \mid \mu_{R}(\overrightarrow{0}, \alpha)=1 \quad \forall \alpha \in \Delta_{n}^{(i)}\right\}, \quad V_{\mu_{R}}=\bigcup_{i \in U_{R}} \Delta_{n}^{(i)} .
$$


Нетрудно убедиться в том, что

$$
2 \leq b_{\chi_{n}}(g) \leq 1+\max \left\{j \mid j \in U_{R}\right\}
$$

Рассмотрим группу $R_{n}^{(2)}=\left\langle\widetilde{S}_{n}, h_{n}\right\rangle$,

$$
h_{n}=h_{1, n} h_{2, n} h_{1, n}, \quad h_{2 m}=1_{2 m} \oplus e_{2 m}, \quad h_{2 m+1}=\left(\begin{array}{cc}
1 & \overrightarrow{1} \\
1 \downarrow & h_{2 m}
\end{array}\right),
$$

где $1_{2 m}-(2 m \times 2 m)$-матрица из единиц.

Для $g \in h_{n} \widetilde{S}_{n}$ несложно показать, что $b_{\chi_{n}}(g)=3$.

Рассмотрим надгруппу $S_{2 m}^{(2)}=\left\langle\widetilde{S}_{2 m}, h_{1,2 m}, h_{2,2 m}\right\rangle$ групп $R_{2 m}^{(1)}, S_{2 m}^{(1)}$. Ее орбиты имеют вид

$$
\Delta_{0}^{(n)}, \quad \Delta_{2 i-1}^{(n)} \cup \Delta_{2 i}^{(n)} \cup \Delta_{2 m-(2 i-1)}^{(n)} \cup \Delta_{2 m-2 i}^{(n)}, \quad i=1, \ldots,\lfloor m / 2\rfloor .
$$

Поэтому $2 \leq b_{\chi_{n}}(g) \leq\lfloor(2 n+4) / 3\rfloor$ для всех $g \in S_{2 m}^{(2)}$; оценка сверху следует из [12].

\section{6. Комбинаторно-геометрические параметры некоторых классов преобразований}

Напомним, что произведение двух циркулянтных матриц - также циркулянтная матрица. Пусть $\alpha=\left(\alpha_{n-1}, \ldots, \alpha_{0}\right), \alpha \in V_{n}$.

Будем говорить, что $(n \times n)$-матрица $a=\left(a_{i j}\right)$ над полем $G F(2)$ является $t$-циркулянтной с порождающим вектором $\alpha$, если $a_{i j}=\alpha_{(n-1-j-i t)}(\bmod n)$ для всех $i, j \in\{0, \ldots, n-1\}$ и некоторого $t \in\{1, \ldots, n-1\}$.

Пусть $\alpha=\left(\alpha_{n-1}, \ldots, \alpha_{0}\right), \beta=\left(\beta_{n-1}, \ldots, \beta_{0}\right)-$ произвольные векторы из $V_{n}$. Матрицы $a=\left(a_{i j}\right)$ и $b=\left(b_{i j}\right)$ соответственно $d_{1}$-циркулянтная и $d_{2}$-циркулянтная с порождающими векторами $\alpha=\left(\alpha_{n-1}, \ldots, \alpha_{0}\right)$ и $\beta=\left(\beta_{n-1}, \ldots, \beta_{0}\right)$. Тогда $a b$ есть $d_{1} d_{2}$-циркулянтная матрица.

Пусть $P$ - поле разложение многочлена $x^{n} \oplus 1$,

$$
c_{n}=\left(c_{i j}\right)=\left(\begin{array}{cccc}
0 & 1 & & \mathbf{0} \\
& \ddots & \ddots & \\
& \mathbf{0} & \ddots & 1 \\
1 & & & 0
\end{array}\right) .
$$

2013, T. 4, № 3, C. 99-129 
Известно, что $u_{c_{n}}(x)=x^{n} \oplus 1$. Кроме того, 1 -циркулянтная матрица $a$ с порождающим вектором $\alpha=\left(\alpha_{n-1}, \ldots, \alpha_{0}\right)$ представима в виде $a=\sum_{k=0}^{n-1} \alpha_{n-1-k} c_{n}^{k}$, а $l$-циркулянтная матрица $a^{(l)}-$ в виде $a^{(l)}=\sum_{k=0}^{m-1} \alpha_{n-1-k} c_{n}^{l k} \bmod n, l \in$ $\in\{1, \ldots, n-1\}$ (см., например, [11]).

Пусть $n=2^{t} d$, где $d$ - простое число, $d>2, t \in \mathbb{N}, f(x)=$ $=\sum_{k=0}^{n-1} \alpha_{n-1-k} x^{k}$. Пусть $\lambda_{0}=1, \lambda_{1}, \ldots, \lambda_{d-1}-$ корни многочлена $x^{d} \oplus 1$, T. e.

$$
x^{n} \oplus 1=\left(x^{d} \oplus 1\right)^{2^{t}}=(x \oplus 1)^{2^{t}} \cdot \prod_{i=1}^{d-1}\left(x \oplus \lambda_{i}\right)^{2^{t}} .
$$

Тогда матрицы $c_{n}$ и $a^{(l)}$ подобны жордановым матрицам

$$
\widetilde{c}_{n}=\left(\begin{array}{ccc}
b_{0} & \mathbf{0} \cdots & \mathbf{0} \\
\mathbf{0} & \ddots & \vdots \\
\vdots & & \mathbf{0} \\
\mathbf{0} & \cdots \mathbf{0} & b_{d-1}
\end{array}\right), \quad \widetilde{a}^{(l)}=f\left(\widetilde{c}_{n}^{l}\right)=\left(\begin{array}{ccc}
\widetilde{b}_{0}^{l} & \mathbf{0} \cdots & \mathbf{0} \\
\mathbf{0} & \ddots & \vdots \\
\vdots & & \mathbf{0} \\
\mathbf{0} & \cdots \mathbf{0} & \widetilde{b}_{d-1}^{l}
\end{array}\right)
$$

соответственно, где $b_{i}-$ жорданова $\left(2^{t} \times 2^{t}\right)$-клетка с корнем $\lambda_{i}$, т. е. $b_{i}=$ $=\operatorname{diag}\left(\lambda_{i}, \ldots, \lambda_{i}\right)$, и $\widetilde{b}_{i}=f\left(b_{i}\right), i=0, \ldots, d-1$. Характеристический многочлен матрицы $\widetilde{a}^{(l)}$ есть $\prod_{i=0}^{d-1}\left(x \oplus f\left(\lambda_{i}^{l}\right)\right)^{2^{t}}$.

В качестве иллюстрации рассмотрим преобразование $g=\left(g_{i j}\right)$ из унитреугольной группы $U T_{n}, n=2^{m}, m \in \mathbb{N}$. В этом случае преобразование $g$ принадлежит силовской 2-подгруппе группы $G L_{n}(2)$ и подобно верхне-треугольной матрице. Положим $g^{d}=\left(g_{i j}^{(d)}\right)$ и $g_{i j}^{(1)}=g_{i j}, d \in \mathbb{N}$.

Утверждение 10. Пусть $m \in \mathbb{N}, m \geq 2, n=2^{m}, g \in U T_{n}, d \in$ $\in\{1, \ldots, m-1\}, i \in\left\{1, \ldots, 2^{m}-1\right\}, j \in\left\{i+1, \ldots, i+2^{d}\right\}$. Тогдa

$$
g_{i j}^{\left(2^{d}\right)}=\left\{\begin{array}{l}
0, \text { если } j<i+2^{d}, \\
g_{i(i+1)} g_{(i+1)(i+2)} \ldots g_{\left(i+2^{d}-1\right)\left(i+2^{d}\right)}, \text { если } j=i+2^{d} .
\end{array}\right.
$$

Доказательство проводится индукцией по $d$, и мы его опускаем.

Из утверждения 10 следует, что если для матрицы $g \in U T_{n}$ найдутся числа $d \in\{1, \ldots, m-1\}, i \in\{1, \ldots, m-d\}$, удовлетворяющие равенству

$$
g_{i(i+1)}=g_{(i+1)(i+2)}=\ldots=g_{\left(i+2^{d}-1\right)\left(i+2^{d}\right)}=1,
$$

то $\operatorname{ord}(g) \geq 2^{d+1}$. В частности, если $d=m-1$, то $\operatorname{ord}(g)=n$. 
Ясно, что $\varepsilon_{0} g=\varepsilon_{0}$ для любой матрицы $g \in U T_{n}$. Значит, $\Gamma_{\left(\overrightarrow{0}, \varepsilon_{0}\right)}(g)=$ $=\left\{\left(\alpha, \alpha \oplus \varepsilon_{0}\right) \mid \alpha \in V_{n}\right\}$. Граф $\bar{\Gamma}_{\left(\overrightarrow{0}, \varepsilon_{0}\right)}(g)$ этого орбитала состоит из $2^{n-1}$ компонент связности, число вершин в каждой компоненте связности равно двум, и $\operatorname{Aut}\left(\bar{\Gamma}_{\left(\overrightarrow{0}_{\varepsilon_{0}}\right)}(g)\right)=S_{2^{n-1}}$ 乙 $S_{2}$.

Отметим, что $g$ имеет систему вложенных инвариантных подпространств $W_{i}=\left\langle\varepsilon_{n-i}, \ldots, \varepsilon_{n-1}\right\rangle, W_{1}<\ldots<W_{n-1}$.

Укажем минимальный многочлен и свойства графов орбиталов группы $C(g)$ при $g \in U T_{n}$. Для этого нам понадобится следующая лемма.

Лемма 6. Пусть $g \in U T_{n}, b=g \oplus e, b^{r}=\left(b_{i j}^{(r)}\right), r \in\{1, \ldots, n-1\}$. Тогда для $i \in\{1, \ldots, n\}, j \in\{1, \ldots, r+i\}$ справедливо равенство

$$
b_{i j}^{(r)}=\left\{\begin{array}{l}
g_{i(i+1)} \ldots g_{(r+i)(r+i+1)}, \text { если } j=r+i, \\
0, \text { если } j<r+i .
\end{array}\right.
$$

Доказательство проводится индукцией по $r$, и мы его опускаем.

Для $i \in\{0, \ldots, n-1\}$ положим

$$
\mathrm{A}_{n}^{(i)}=\left\{(\underbrace{0, \ldots, 0}_{n-i-1}, 1, \alpha_{i-1}, \ldots, \alpha_{0}) \mid\left(\alpha_{i-1}, \ldots, \alpha_{0}\right) \in V_{i}\right\} .
$$

Утверждение 11. Пусть $n \geq 2, r \in\{1, \ldots, n\} u g \in U T_{n}$.

1. Равенство $m_{g}(x)=u_{g}(x)=(x \oplus 1)^{n}$ выполняется тогда и только тогда, когда $g_{12}=\ldots=g_{(n-1) n}=1$.

2. Если $g_{12} g_{23} \cdot \ldots \cdot g_{(n-1) n}=0$, то все графы орбиталов группы $C(g)$ не связньл.

3. Пусть $g_{12}=\ldots=g_{(n-1) n}=1$. Тогда $m_{\gamma, g}(x)=(x \oplus 1)^{i+1}$ для любого вектора $\gamma \in \mathrm{A}_{n}^{(i)}, i \in\{0, \ldots, n-1\}$.

Доказательство. Положим $b=g \oplus e_{n}, b^{r}=\left(b_{i j}^{r}\right), r \in\{1, \ldots, n\}$. Из леммы 6 следуют пп. 1 и 2.

3. Пусть $i \in\{0, \ldots, n-1\}, \gamma=\left(\gamma_{n-1}, \ldots, \gamma_{0}\right) \in \mathrm{A}_{n}^{(i)}$ и $\gamma^{\prime}=\gamma b^{c}$, $c \in\{0, \ldots, n-1\}$. Для $j \in\{0, \ldots, n-1\}$ справедливо равенство

$$
\widetilde{\gamma}_{n-j-1}=\sum_{k=1}^{n} \oplus \gamma_{n-k} b_{k(j+1)}^{(c)}=\sum_{k=n-i}^{j-c+1} \oplus \gamma_{n-k} b_{k(j+1)}^{(c)},
$$

2013, T. 4, № 3, C. 99-129 
причем $\widetilde{\gamma}_{n-j-1}=0$ при $j-c+1<n-i$. Кроме того, $\widetilde{\gamma}=\overrightarrow{0}$ при $i<c$ (так как $n-1<n-i+c-1)$.

Если $i=c$, то при $j<n-1$ имеем $\widetilde{\gamma}_{n-j-1}=0$. Если же $j=n-1$, то $\widetilde{\gamma}_{0}=\gamma_{i} b_{(n-i) n}^{(i)}=1$, так как $\gamma_{i}=1$ по определению, а равенство $b_{(n-i) n}^{(i)}=1$ следует из леммы 6. Значит, $\gamma(g \oplus e)^{i} \neq \overrightarrow{0}$, а $\gamma(g \oplus e)^{i+1}=\overrightarrow{0}$.

Таким образом, $m_{\gamma, g}(x)=(x \oplus 1)^{i+1}$ для любого вектора $\gamma \in \mathrm{A}_{n}^{(i)}$. Утверждение доказано.

Для $a \in G L_{n}$ положим

$$
\begin{gathered}
\mathrm{B}_{n}^{(0)}(a)=\left\{\beta \in V_{n}^{\times} \mid \beta a=\beta\right\}, \\
\mathrm{B}_{n}^{(t)}(a)=\left\{\beta \in V_{n}^{\times} \backslash\left(\bigcup_{j=0}^{t-1} \mathrm{~B}_{n}^{(j)}(a)\right) \mid \beta a^{2^{t}}=\beta\right\}, \quad t=1, \ldots, m .
\end{gathered}
$$

Отметим, что при $a_{12}=\ldots=a_{(n-1) n}=1$ из утверждения 10 следуют равенства ord $(a)=n$ и $\mathrm{B}_{n}^{(0)}(a)=\left\{\varepsilon_{0}\right\}$.

Далее нам понадобится следующий технический результат. Тогда

Лемма 7. Пусть $n \in \mathbb{N}, n \geq 2, g \in U T_{n} u g_{12}=\ldots=g_{(n-1) n}=1$.

$$
\mathrm{B}_{n}^{(0)}=\mathrm{A}_{n}^{(0)}, \quad \mathrm{B}_{n}^{(t)}=\bigcup_{j=2^{t-1}}^{2^{t}-1} \mathrm{~A}_{n}^{(t)} \text { для любого } t \in\{1, \ldots, m\}
$$

Доказательство проводится индукцией по $t$, и мы его опускаем.

Для $i \in\{0, \ldots, n-1\}$ и $g \in U T_{n}$ обозначим через $\mathrm{A}_{n}^{(i)}(g)$ множество всех таких векторов $\gamma \in \mathrm{A}_{n}^{(i)}$, что $\Gamma_{(\overrightarrow{0}, \gamma)}(g) \neq \Gamma_{(\overrightarrow{0}, \beta)}(g)$ для каждого $\beta \in$ $\in \Gamma^{(i)}(g) \backslash\{\gamma\}$. Положим $\Omega_{n}^{(i)}(g)=\left\{\bar{\Gamma}_{(\overrightarrow{0}, \gamma)}(g) \mid \gamma \in \mathrm{A}_{n}^{(i)}(g)\right\}$.

Опишем классы изоморфных графов среди графов орбиталов группы $C(g)$ для $g \in U T_{n}$.

Следствие 3. Пусть $n \geq 2, g \in U T_{n}, i \in\{1, \ldots, n-1\}$. Тогда все графы из множества $\Omega_{n}^{(i)}(g)$ изоморфны, $\left|\Omega_{n}^{(i)}(g)\right|=2^{i-\left\lceil\log _{2}(i+1)\right\rceil}$. Если $i=$ $=2^{t}-1 t \in\left\{1, \ldots,\left\lfloor\log _{2} n\right\rfloor+1\right\}$, то компоненты связности графа $\bar{\Gamma}_{(\overrightarrow{0}, \gamma)}(g)$ изоморфны графу Хемминга на пространстве $V_{2^{t}}$.

Доказательство. Из утверждения 5 и утверждения 11 следует, что все графы из множества $\Omega_{n}^{(i)}(g)$ изоморфны. Согласно лемме 7 для любого 
вектора $\gamma \in \mathrm{A}_{n}^{(i)}$ справедливо равенство $\left|\gamma^{\langle g\rangle}\right|=2^{\left\lceil\log _{2}(i+1)\right\rceil+1}$. Так как каждому вектору $\gamma \in \mathrm{A}_{n}^{(i)}$ соответствует граф $\bar{\Gamma}_{(\overrightarrow{0}, \gamma)}(g)$ и все графы множества $\mathrm{A}_{n}^{(i)}(g)$ изоморфны, то и все графы $\bar{\Gamma}_{(\overrightarrow{0}, \gamma)}(g), \gamma \in \mathrm{A}_{n}^{(i)}$, изоморфны. Кроме того, из леммы 7 следует, что $\mathrm{A}_{n}^{(i)} g=\mathrm{A}_{n}^{(i)}$. Очевидно, что если $\gamma_{2} \in \gamma_{1}^{\langle g\rangle}$ для $\gamma_{1}, \gamma_{2} \in A_{n}^{(i)}(g)$, то $\bar{\Gamma}_{\left(\overrightarrow{0}, \gamma_{1}\right)}(g)=\bar{\Gamma}_{\left(\overrightarrow{0}, \gamma_{2}\right)}(g)$. Таким образом,

$$
\left|\Omega_{n}^{(i)}(g)\right|=\left|\mathrm{A}_{n}^{(i)}\right| / 2^{\left\lceil\log _{2}(i+1)\right\rceil+1}=2^{i-\left\lceil\log _{2}(i+1)\right\rceil} .
$$

Если $i=2^{t}-1$, то $m_{\gamma, g}(x)=(x \oplus 1)^{2^{t}}=x^{2^{t}} \oplus 1$ для любого $\gamma \in \mathrm{A}_{n}^{(i)}$. Если $2^{t}<n$, то граф $\bar{\Gamma}_{(\overrightarrow{0}, \gamma)}(g)$ не связен, и из теоремы 1 получаем, что все его компоненты связности изоморфны графу Хемминга на пространстве $V_{2^{t}}$. Значит, их метрики изоморфны метрике $\chi_{2^{t}}$. Если же $2^{t}=n$, то граф $\bar{\Gamma}_{(\overrightarrow{0}, \gamma)}(g)$ связен и изоморфен графу Хемминга с метрикой $\chi_{n}$. Следствие доказано.

Приведем примеры метрик и других характеристик графов орбиталов группы $C(g)$ для циркулянтных матриц и матриц некоторых блочных шифрсистем.

\section{Примеры}

1. У циркулянтной матрицы

$$
a_{1}=\left[\begin{array}{llll}
0 & 1 & 1 & 1 \\
1 & 1 & 1 & 0 \\
1 & 1 & 0 & 1 \\
1 & 0 & 1 & 1
\end{array}\right]
$$

характеристический многочлен есть $u_{a_{1}}(x)=(x \oplus 1)^{4}$, минимальный многочлен $m_{a_{1}}(x)=(x \oplus 1)^{2}$. Матрица $a_{1}$ приводима, $a_{1}^{2}=e$. Все графы орбиталов не связны. Кроме того, $\left(\alpha_{3}, \alpha_{2}, \alpha_{1}, \alpha_{0}\right) a_{1}=\left(\alpha_{3}, \alpha_{2}, \alpha_{1}, \alpha_{0}\right)$ тогда и только тогда, когда $\alpha_{3} \oplus \alpha_{2} \oplus \alpha_{1} \oplus \alpha_{0}=0$. Пусть $B_{4}-$ множество всех векторов четного веса из $V_{4}^{\times}$, т. е. $B=\Delta_{2}^{(4)} \cup \Delta_{4}^{(4)}$.

В зависимости от вектора $\gamma \in V_{4}^{\times}$возможны два вида орбиталов:

$$
\Gamma_{(\overrightarrow{0}, \gamma)}(g)=\left\{\begin{array}{l}
\left\{(\alpha, \alpha \oplus \theta) \mid \alpha \in V_{4}, \theta \in\left\{\gamma, \gamma^{a_{1}}\right\}\right\}, \text { если } \gamma \in V_{4}^{\times} \backslash B_{4}, \\
\left\{(\alpha, \alpha \oplus \gamma) \mid \alpha \in V_{4}\right\}, \text { если } \gamma \in B_{4} .
\end{array}\right.
$$

Таким образом, если $\gamma \in B_{4}$, то граф $\bar{\Gamma}_{(\overrightarrow{0}, \gamma)}(g)$ состоит из $2^{3}$ компонент связности, число вершин у каждой компоненты связности равно двум 
и Aut $\left(\bar{\Gamma}_{(\overrightarrow{0}, \gamma)}(g)\right)=S_{2^{3}} \succ S_{2}$. Если же $\gamma \in V_{4}^{\times} \backslash B_{4}$, то граф $\bar{\Gamma}_{(\overrightarrow{0}, \gamma)}(g)$ состоит из $2^{2}$ компонент связности, число вершин у каждой компоненты связности равно 4. Метрика графа каждой компоненты связности изоморфна метрике Хемминга $\chi_{2}$. Кроме того, Aut $\left(\bar{\Gamma}_{(\overrightarrow{0}, \gamma)}(g)\right)=S_{2^{2}} \succ D_{4}$, где $D_{4}-$ группа диэдра.

2. Согласно [13] матрица

$$
a_{2}=\left[\begin{array}{llllllll}
0 & 1 & 1 & 1 & 1 & 1 & 1 & 0 \\
1 & 0 & 1 & 1 & 0 & 1 & 1 & 1 \\
1 & 1 & 0 & 1 & 1 & 0 & 1 & 1 \\
1 & 1 & 1 & 0 & 1 & 1 & 0 & 1 \\
1 & 1 & 0 & 1 & 1 & 1 & 0 & 0 \\
1 & 1 & 1 & 0 & 0 & 1 & 1 & 0 \\
0 & 1 & 1 & 1 & 0 & 0 & 1 & 1 \\
1 & 0 & 1 & 1 & 1 & 0 & 0 & 1
\end{array}\right]
$$

обладает хорошими рассеивающими свойствами. Она используется в алгоритме блочного шифрования Е2. Ее коэффициент рассеивания равен 5 и лишь на единицу меньше максимального для $(0,1)$-матриц (см. [12]).

Ее характеристический многочлен равен $\left(x^{2} \oplus x \oplus 1\right)^{4}$, ord $\left(a_{2}\right)=12$. Непосредственные вычисления показали, что число попарно различных графов орбиталов равно 23. Причем имеются: 1) 20 графов орбиталов, которым соответствует орбита преобразования $a_{2}$ длины 12 ; 2) 2 изоморфных графа орбиталов, которым соответствует орбита преобразования $a_{2}$ длины 6; 3) один граф орбитала, которому соответствует орбита преобразования $a_{2}$ длиной 3.

3. У матрицы

$$
a_{3}=\left[\begin{array}{llllllll}
1 & 1 & 1 & 0 & 1 & 0 & 0 & 1 \\
0 & 1 & 1 & 1 & 1 & 1 & 0 & 0 \\
1 & 0 & 1 & 1 & 0 & 1 & 1 & 0 \\
1 & 1 & 0 & 1 & 0 & 0 & 1 & 1 \\
0 & 1 & 1 & 1 & 0 & 1 & 1 & 1 \\
1 & 0 & 1 & 1 & 1 & 0 & 1 & 1 \\
1 & 1 & 0 & 1 & 1 & 1 & 0 & 1 \\
1 & 1 & 1 & 0 & 1 & 1 & 1 & 0
\end{array}\right]
$$

алгоритма блочного шифрования Camellia характеристический и минимальный многочлен равны $(x \oplus 1)^{8}$. Выполняется равенство $a_{3}^{8}=e$. 
Положим

$$
\begin{gathered}
B_{8}^{(0)}=\left\{\alpha \in V_{8}^{\times} \mid \alpha a_{3}=\alpha\right\}, \\
B_{8}^{(1)}=\left\{\alpha \in V_{8}^{\times} \backslash B_{8}^{(0)} \mid \alpha a_{3}^{2}=\alpha\right\}, \\
B_{8}^{(2)}=\left\{\alpha \in V_{8}^{\times} \backslash\left(B_{8}^{(0)} \cup B_{8}^{(1)}\right) \mid \alpha a_{3}^{4}=\alpha\right\} .
\end{gathered}
$$

В зависимости от вектора $\gamma \in V_{8}^{\times}$возможны следующие классы орбиталов:

$$
\begin{aligned}
& \Gamma_{(\overrightarrow{0}, \gamma)}(g)= \\
& =\left\{\begin{array}{l}
\left\{(\alpha, \alpha \oplus \theta) \mid \alpha \in V_{8}, \theta \in \gamma^{\left\langle a_{3}\right\rangle}\right\}, \text { если } \gamma \in V_{8}^{\times} \backslash\left(B_{8}^{(0)} \cup B_{8}^{(1)} \cup B_{8}^{(2)}\right), \\
\left\{(\alpha, \alpha \oplus \theta) \mid \alpha \in V_{8}, \theta \in\left\{\gamma, \gamma^{a_{3}}, \gamma^{a_{3}^{2}}, \gamma^{a_{3}^{3}}\right\}\right\}, \text { если } \gamma \in B_{8}^{(2)}, \\
\left\{(\alpha, \alpha \oplus \theta) \mid \alpha \in V_{8}, \theta \in\left\{\gamma, \gamma^{a_{3}}\right\}\right\}, \text { если } \gamma \in B_{8}^{(1)}, \\
\left\{(\alpha, \alpha \oplus \gamma) \mid \alpha \in V_{8}\right\}, \text { если } \gamma \in B_{8}^{(0)} .
\end{array}\right.
\end{aligned}
$$

Таким образом, если $\gamma \in B_{8}^{(0)}$, то граф $\bar{\Gamma}_{(\overrightarrow{0}, \gamma)}(g)$ состоит из $2^{7}$ компонент связности из двух вершин каждая и Aut $\left(\bar{\Gamma}_{(\overrightarrow{0}, \gamma)}(g)\right)=S_{2^{7}}$ 2 $S_{2}$. Если же $\gamma \in B_{8}^{(1)}$, то граф $\bar{\Gamma}_{(\overrightarrow{0}, \gamma)}(g)$ состоит из $2^{6}$ изоморфных компонент связности из четырех вершин каждая. Метрика графа каждой компоненты связности изоморфна метрике Хемминга $\chi_{2}$. Кроме того, Aut $\left(\Gamma_{(\overrightarrow{0}, \gamma)}(g)\right)=S_{2^{6}} \prec D_{4}$, где $D_{4}-$ группа диэдра.

Поскольку $a_{3}^{8}=e$, то $a_{3} \in U T_{n}$. В силу следствия 3 :

- Для $\gamma \in \mathrm{A}_{8}^{(7)}$ метрика графа $\bar{\Gamma}_{(\overrightarrow{0}, \gamma)}(g)$ изоморфна метрике Хемминга, и $\operatorname{Aut}\left(\bar{\Gamma}_{(\overrightarrow{0}, \gamma)}(g)\right)=S_{2} \uparrow S_{8}$. Имеется 16 графов орбиталов, изоморфных графу Хемминга.

- Для $\gamma \in \mathrm{A}_{8}^{(6)}$ имеется 8 графов орбиталов, состоящих из двух компонент связности, у которых число линейно независимых векторов в множестве $\gamma^{\left\langle a_{3}\right\rangle}$ равно 7.

- Для $\gamma \in \mathrm{A}_{8}^{(5)}$ имеется 4 изоморфных графа орбиталов, состоящих из четырех компонент связности, у которых число линейно независимых векторов в множестве $\gamma^{\left\langle a_{3}\right\rangle}$ равно 6 .

- Для $\gamma \in \mathrm{A}_{8}^{(4)}$ имеется два изоморфных графа орбиталов, состоящих из 8 компонент связности, у которых число линейно независимых векторов в множестве $\gamma^{\left\langle a_{3}\right\rangle}$ равно 5. 
4. У матрицы Адамара типа 2

$$
a_{4}=\left[\begin{array}{llllllll}
1 & 1 & 1 & 1 & 1 & 0 & 0 & 0 \\
1 & 1 & 1 & 1 & 0 & 1 & 0 & 0 \\
1 & 1 & 1 & 1 & 0 & 0 & 1 & 0 \\
1 & 1 & 1 & 1 & 0 & 0 & 0 & 1 \\
1 & 0 & 0 & 0 & 1 & 1 & 1 & 1 \\
0 & 1 & 0 & 0 & 1 & 1 & 1 & 1 \\
0 & 0 & 1 & 0 & 1 & 1 & 1 & 1 \\
0 & 0 & 0 & 1 & 1 & 1 & 1 & 1
\end{array}\right]
$$

характеристический многочлен есть $u_{a_{4}}(x)=(x \oplus 1)^{8}$. Матрица $a_{4}$ приводима, $a_{4}^{2}=e$. Все графы орбиталов не связны.

Положим $B_{8}^{(0)}=\left\{\alpha \in V_{8}^{\times} \mid \alpha a_{4}=\alpha\right\}$. Если $\gamma \in B_{8}^{(0)}$, то граф орбитала состоит из $2^{7}$ компонент связности, число вершин в каждой компоненте связности равно двум, и Aut $\left(\bar{\Gamma}_{(\overrightarrow{0}, \gamma)}(g)\right)=S_{2^{7}}$ 乙 $S_{2}$. Если же $\gamma \in V_{8}^{\times} \backslash B_{8}^{(0)}$, то граф орбитала состоит из $2^{6}$ компонент связности, число вершин в каждой компоненте связности равно 4. Метрика графа каждой компоненты связности изоморфна метрике Хемминга $\chi_{2}$. Кроме того, Aut $\left(\Gamma_{(\overrightarrow{0}, \gamma)}(g)\right)=S_{2^{6}} D_{4}$, где $D_{4}$ - группа диэдра.

5. У циркулянтной матрицы

$$
a_{5}=\left[\begin{array}{llllllll}
1 & 1 & 0 & 1 & 0 & 1 & 0 & 1 \\
1 & 0 & 1 & 0 & 1 & 0 & 1 & 1 \\
0 & 1 & 0 & 1 & 0 & 1 & 1 & 1 \\
1 & 0 & 1 & 0 & 1 & 1 & 1 & 0 \\
0 & 1 & 0 & 1 & 1 & 1 & 0 & 1 \\
1 & 0 & 1 & 1 & 1 & 0 & 1 & 0 \\
0 & 1 & 1 & 1 & 0 & 1 & 0 & 1 \\
1 & 1 & 1 & 0 & 1 & 0 & 1 & 0
\end{array}\right]
$$

характеристический многочлен есть $u_{a_{5}}(x)=(x \oplus 1)^{8}$. Матрица $a_{5}$ приводима, $a_{5}^{2}=e$. Ситуация в этом случае описывается так же, как в случае 3 .

\section{Список литературы}

1. Погорелов Б. А. Основы теории групп подстановок. - М.: 1986.

2. Глухов М. М., Елизаров В.П., Нечаев А.А. Алгебра. Т. 2. - М.: Гелиос APB, 2003. 
3. Godsil C., Royle G. Algebraic Graph Theory. - Springer-Verlag, 2001.

4. Погорелов Б.А., Пудовкина М.А. Свойства графов орбиталов надгрупп группы Джевонса // Математические вопросы криптографии. - 2010. Т. 1. Вып. 1 - С. 55-83.

5. Погорелов Б.А. Подметрики метрики Хемминга и теорема А.А. Маркова // В сб. : Труды по дискретной математике. Т. 9. - М.: ФИЗМАТЛИТ, 2006. - C. 190-219.

6. Погорелов Б. А., Пудовкина М. А. Подметрики Хемминга и их группы изометрий // В сб. : Труды по дискретной математике. - М.: ФИЗМАТЛИТ, 2008. - Т. 11. Вып. 2. - С.147-191.

7. Deza M., Deza E. Encyclopedia of Distances. - Springer-Verlag, Berlin Heidelberg, 2009.

8. Баннаи Э., Ито Т. Алгебраическая комбинаторика. - М.: Мир, 1987.

9. Погорелов Б.А., Пудовкина М.А. Натуральные метрики и их свойства. Ч. 1. Подметрики и надметрики // Математические вопросы криптографии. - 2011. - Т. 2. Вып. 4. - С. 47-76.

10. Погорелов Б.А., Пудовкина М.А. Натуральные метрики и их свойства. Ч. 2. Случай метрик Хемминга // Математические вопросы криптографии. - 2012. - Т. 3. Вып. 1. - С. 71-95.

11. Сачков В. Н., Тараканов В. Е. Комбинаторика неотрицательных матриц. М. : ТВП, 2000.

12. Choy J., Khoo K. New applications of differential bounds of the SDS structure // ISC'08. - 2008.

13. Kanda M., Moriai S., Aoki K., Ueda H., Takashima Y., Ohta K., Matsumoto T. E2 - A New 128-bit Block Cipher // IEICE Trans. Fund. - Spec. Sect. on Cryptography and Inf. Security. - 2000. - V. E83-A , No. 1. - P. 48-59.

14. Daemen J. Cipher and hash function design strategies based on linear and differential cryptanalysis. - PhD. - K. U. Leuven, 1995.

15. Глухов М.M. О рассеивающих линейных преобразованиях для блочных шифрсистем // Математические вопросы криптографии. - 2011. - Т. 2. Вып. 2. - С. 5-40. 